IJMMS 27:10 (2001) 599-620

PII. S0161171201006792

http://ijmms.hindawi.com

(c) Hindawi Publishing Corp.

\title{
BLASCHKE INDUCTIVE LIMITS OF UNIFORM ALGEBRAS
}

\section{S. A. GRIGORYAN and T. V. TONEV}

(Received 16 February 2001)

\begin{abstract}
We consider and study Blaschke inductive limit algebras $A(b)$, defined as inductive limits of disc algebras $A(D)$ linked by a sequence $b=\left\{B_{k}\right\}_{k=1}^{\infty}$ of finite Blaschke products. It is well known that big $G$-disc algebras $A_{G}$ over compact abelian groups $G$ with ordered duals $\Gamma=\hat{G} \subset \mathbb{Q}$ can be expressed as Blaschke inductive limit algebras. Any Blaschke inductive limit algebra $A(b)$ is a maximal and Dirichlet uniform algebra. Its Shilov boundary $\partial A(b)$ is a compact abelian group with dual group that is a subgroup of $\mathbb{Q}$. It is shown that a big $G$-disc algebra $A_{G}$ over a group $G$ with ordered dual $\hat{G} \subset \mathbb{R}$ is a Blaschke inductive limit algebra if and only if $\hat{G} \subset \mathbb{Q}$. The local structure of the maximal ideal space and the set of one-point Gleason parts of a Blaschke inductive limit algebra differ drastically from the ones of a big $G$-disc algebra. These differences are utilized to construct examples of Blaschke inductive limit algebras that are not big $G$-disc algebras. A necessary and sufficient condition for a Blaschke inductive limit algebra to be isometrically isomorphic to a big $G$-disc algebra is found. We consider also inductive limits $H^{\infty}(I)$ of algebras $H^{\infty}$, linked by a sequence $I=\left\{I_{k}\right\}_{k=1}^{\infty}$ of inner functions, and prove a version of the corona theorem with estimates for it. The algebra $H^{\infty}(I)$ generalizes the algebra of bounded hyper-analytic functions on an open big $G$-disc, introduced previously by Tonev.
\end{abstract}

2000 Mathematics Subject Classification. 46J15, 46J20, 30H05.

1. Introduction. Let $\mathbb{T}=\{z \in \mathbb{C}:|z|=1\}$ denote the unit circle and let $\mathbb{D}=\{z \in \mathbb{C}$ : $|z| \leq 1\}$ be the closed unit disc in $\mathbb{C}$. Consider an inductive sequence

$$
A\left(\mathbb{T}_{1}\right) \stackrel{i_{1}^{2}}{\longrightarrow} A\left(\mathbb{T}_{2}\right) \stackrel{i_{2}^{3}}{\longrightarrow} A\left(\mathbb{T}_{3}\right) \stackrel{i_{3}^{4}}{\longrightarrow} \cdots
$$

of disc algebras $A\left(\mathbb{T}_{k}\right)=A(\mathbb{T})$ linked by homomorphisms $i_{k}^{k+1}: A\left(\mathbb{T}_{k}\right) \rightarrow A\left(\mathbb{T}_{k+1}\right)$. Every conjugate mapping $\left(i_{k}^{k+1}\right)^{*}: \mu_{k} \leftarrow \mathcal{M}_{k+1}$ maps the maximal ideal space $\mu_{k+1} \approx \overline{\mathbb{D}}$ of $A\left(\mathbb{T}_{k+1}\right)$ into the maximal ideal space $\mu_{k} \approx \overline{\mathbb{D}}$ of $A\left(\mathbb{T}_{k}\right)$. Since $i_{k}^{k+1}(f)=f \circ\left(i_{k}^{k+1}\right)^{*} \in$ $A\left(\mathbb{T}_{k+1}\right)$ for every $f \in A\left(\mathbb{T}_{k}\right)$, the mapping $\left(i_{k}^{k+1}\right)^{*}$ is an analytic function preserving the unit disc. The inverse limit

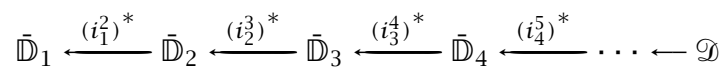

is the maximal ideal space of the inductive limit algebra

$$
\left[\underset{k \rightarrow \infty}{\lim _{k}}\left\{A\left(\mathbb{\mathbb { T }}_{k}\right), i_{k}^{k+1}\right\}\right],
$$

where the closure is taken in $C(\mathscr{D})$. In general, the mappings $\left(i_{k}^{k+1}\right)^{*}$ are not obliged to map the unit circle $\mathbb{T}_{k+1}$ onto itself. The most interesting situations, though, are 
the ones when they do, and this is what we will assume in the sequel. In effect, the mappings $\left(i_{k}^{k+1}\right)^{*}$ become finite Blaschke products

$$
B_{k}(z)=e^{i \theta} \prod_{s=1}^{n_{k}}\left(\frac{z-z_{s, k}}{1-\bar{z}_{s, k} z}\right), \quad 0<\left|z_{s, k}\right|<1
$$

on $\overline{\mathbb{D}}$. The inductive limit algebra $\left[\mathrm{lim}_{k \rightarrow \infty}\left\{A\left(\mathbb{T}_{k}\right), i_{k}^{k+1}\right\}\right]$ in this case is called a Blaschke inductive limit algebra. Note that all algebras $i_{k}^{k+1}\left(A\left(\mathbb{\mathbb { T }}_{k}\right)\right)$ are algebraic extensions of the disc algebra that are isometrically isomorphic to the disc algebra itself. Indeed, let $A$ be an algebra and let $A[x]$ be the algebra of polynomials in $x$ over $A$. For a given unital polynomial $p(x)=x^{n}+a_{1} x^{n-1}+\cdots+a_{n}, a_{j} \in A$ in $A[x]$ the set $p(x) A[x]$ is an ideal in the algebra $A[x]$. Recall that the algebraic extension of $A$ by $p(x)$ is the algebra

$$
A_{p}=A[x] /(p(x) A[x]) .
$$

$A_{p}$ is isometrically isomorphic to $A(\mathbb{T})$ if and only if the diagram

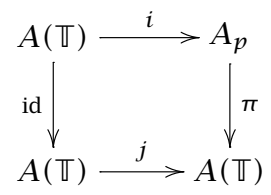

is commutative, where $i$ is the natural embedding $i: A(\mathbb{T}) \rightarrow A_{p}$, and $\pi: A_{p} \rightarrow A(\mathbb{T})$ is an isomorphism. In this case the homomorphism $j=\pi \circ i: A(\mathbb{T}) \rightarrow A(\mathbb{T})$ coincides with the composition operator $C_{B}=f \circ B$ defined by a finite Blaschke product $B$, that is, $(j(f(z)))=\left(C_{B}(f)\right)(z)=f(B(z))$.

Let $G$ be a compact abelian group, whose dual group $\hat{G}$ is isomorphic to a subgroup $\Gamma$ of $\mathbb{R}$. Denote by $A_{G}$ the big $G$-disc algebra generated by $\Gamma$, that is, $A_{G}$ is the uniform algebra on $G$ generated by the semigroup of characters $\left\{x^{a} \in \hat{G}: a \in \Gamma_{+}\right\}$, where $\Gamma_{+}=\{a \in \Gamma: a \geq 0\}$ is the positive part of $\Gamma$. The elements in $A_{G}$ are referred to as generalized $G$-analytic functions on $G$. In Section 2 we review some results on finite Blaschke products and generalized $G$-analytic functions. In Section 3, we show that Blaschke inductive limit algebras share many properties with big $G$-disc algebras. We give also necessary and sufficient conditions on a group $\Gamma \subset \mathbb{R}$ so that the big $G$-disc algebra $A_{G}, G=\widehat{\Gamma}$ can be expressed as the inductive limit of a Blaschke sequence of (algebraic extensions of) disc algebras. In Section 4, we study annulus type Blaschke inductive limit algebras. The local structure of Blaschke inductive limit algebras is studied in Section 5. We construct Blaschke inductive sequences of disc algebras whose limits are not big $G$-disc algebras. In Section 6, we describe the one-point Gleason parts in the maximal ideal space of a Blaschke inductive limit algebra. This description plays a crucial role in Section 7, where we find necessary and sufficient conditions for a Blaschke inductive limit algebra to be expressed as a big $G$-disc algebra. In Section 8, we consider inductive limits of algebras $H^{\infty}$ that are linked by inner functions, and prove the corona theorem for them. 
2. Preliminaries. Here we state several basic results on finite Blaschke products and generalized $G$-analytic functions, we will need further. Given a uniform algebra $A, M_{A}$ and $\partial A$ will denote the maximal ideal space and Shilov boundary of $A$ correspondingly. Any homomorphism $\varphi: A \rightarrow B$ between two uniform algebras naturally generates a conjugate map $\varphi^{*}: \mu_{A} \leftarrow \mu_{B}$ between their maximal ideal spaces. If, in addition, $\varphi$ is an isometry, that is, if

$$
\|\varphi(g)\|_{B}=\|g\|_{A}
$$

for every $g \in A$, then $\varphi$ is called an embedding of $A$ into $B$.

LEMMA 2.1. Let $A$ and $B$ be uniform algebras. A homomorphism $\varphi: A \rightarrow B$ generates an embedding of $A$ into $B$ if and only if $\varphi^{*}(\partial B) \supset \partial A$.

Proof. Note that for every $g \in A$ we have

$$
\max _{m \in \varphi^{*}(\partial B)}|m(g)|=\max _{s \in \partial B}\left|\left(\varphi^{*}(s)\right)(g)\right|=\max _{s \in \partial B}|s(\varphi(g))|=\|\varphi(g)\|_{B} .
$$

If $\varphi^{*}(\partial B) \supset \partial A$, then $\|g\|_{A}=\max _{m \in \partial A}|m(g)|=\|\varphi(g)\|_{B}$. Hence $\varphi$ is an isometry. On the other hand, if $\varphi$ is an isometry, then

$$
\max _{m \in \varphi^{*}(\partial B)}|m(g)|=\|\varphi(g)\|_{B}=\|(g)\|_{A}
$$

implies that the set $\varphi^{*}(\partial B)$ is a boundary for $A$. Therefore $\varphi^{*}(\partial B) \supset \partial A$.

Note that every embedding $j: A(\mathbb{T}) \rightarrow A(\mathbb{T})$ of the disc algebra into itself generates an isometric isomorphism between $A(\mathbb{T})$ and $j(A(\mathbb{T}))$. Hence $j^{*}: \mu_{j(A(\mathbb{T}))} \rightarrow \mu_{A(\mathbb{T})} \cong \overline{\mathbb{D}}$ is a homeomorphism and $j^{*} \partial(j(A(\mathbb{T})))=\partial A(\mathbb{T})=\mathbb{T}$. If, in addition, $\mu_{j(A(\mathbb{T}))}=\overline{\mathbb{D}}$ and $\partial(j(A(\mathbb{T})))=\mathbb{T}$, then $j^{*}(\mathbb{T})=\mathbb{T}$, and hence the function $j^{*}$ is a finite Blaschke product (see [6], Chapter I, 2). Consequently, for any isometry $j: A(\mathbb{T}) \rightarrow A(\mathbb{T})$ with the above properties there is a Blaschke product

$$
B(z)=e^{i \theta} \prod_{k=1}^{n}\left(\frac{z-z_{k}}{1-\bar{z}_{k} z}\right), \quad 0<\left|z_{k}\right|<1,
$$

such that

$$
(j \circ f)(z)=f \circ j^{*}(z)=f(B(z)) \quad \forall f \in A(\mathbb{T}) .
$$

Recall that $z_{0} \in \mathbb{D}$ is a critical point for $B$ if $B^{\prime}\left(z_{0}\right)=0$, that is, if $\operatorname{card}\left(B^{-1}\left(z_{0}\right)\right)<$ ord $B$. By the Brower's fixed point theorem, $B$ always has a fixed point, that is, $B\left(z_{0}\right)=$ $z_{0}$ for some $z_{0} \in \overline{\mathbb{D}}$. If the order of $B$ is greater than 1 then by the Schwartz lemma the fixed point of $B$ is unique.

We will need in the sequel the following result, which is probably well known.

LEMMA 2.2. If $B$ is a finite Blaschke product with a single critical point $z_{0} \in \mathbb{D}$, then

$$
B(z)=\frac{\tau_{\theta}(z)^{m}+B\left(z_{0}\right)}{1+\overline{B\left(z_{0}\right)} \tau_{\theta}(z)^{m}},
$$

where $m=\operatorname{ord} B$ and $\tau_{\theta}=e^{i \theta}\left(z-z_{0}\right) /\left(1-\bar{z}_{0} z\right)$ for some $\theta, 0 \leq \theta<2 \pi$. 
Proof. The restriction of $B$ on $\mathbb{D} \backslash\left\{z_{0}\right\}$ generates a holomorphic covering from $\mathbb{D} \backslash\left\{z_{0}\right\}$ onto $\mathbb{D} \backslash\left\{B\left(z_{0}\right)\right\}$. If $\varphi(z)=\left(z-B\left(z_{0}\right)\right) /\left(1-\overline{B\left(z_{0}\right)} z\right)$, then the composition $\varphi \circ B$ generates an unramified $m$-sheeted holomorphic covering from $\mathbb{D} \backslash\left\{z_{0}\right\}$ onto $\mathbb{D} \backslash\{0\}$. Consequently, there exists a biholomorphic map $\sigma: \mathbb{D} \backslash\left\{z_{0}\right\} \rightarrow \mathbb{D} \backslash\{0\}$, such that $(\varphi \circ B)(z)=\sigma(z)^{m}$ (cf. [6]). Clearly, $\sigma=\tau_{\theta}$ for some $\theta: 0 \leq \theta<2 \pi$, that is, $\varphi(B(z))=\left(\tau_{\theta}(z)\right)^{m}$. Hence

$$
B(z)=\varphi^{-1}\left(\tau_{\theta}(z)\right)^{m}=\frac{\tau_{\theta}(z)^{m}+B\left(z_{0}\right)}{1+\overline{B\left(z_{0}\right)} \tau_{\theta}(z)^{m}} .
$$

Let $G$ be a compact abelian group. We assume that its dual $\hat{G}$ is isomorphic to a subgroup $\Gamma$ of $\mathbb{R}$. The big $G$-disc $\bar{\Delta}_{G}$ over $G$ is the compact set obtained from the Cartesian product $[0,1] \times G$ by identifying the points in the fiber $\{0\} \times G$. The group $G \approx\{1\} \times G \subset \bar{\Delta}_{G}$ is the topological boundary of $\bar{\Delta}_{G}$. If $\Gamma=\mathbb{Z}$, then $\hat{G}=\widehat{\mathbb{Z}}=\mathbb{T}$, and the big $G$-disc algebra $A_{G}$ coincides with the classical disc algebra. We list here some of the basic properties of $A_{G}$.

(a) $A_{G}$ is a maximal Dirichlet algebra.

(b) The maximal ideal space $\mu_{A_{G}}$ of $A_{G}$ is homeomorphic to the closed big disc $\bar{\Delta}_{G}$.

(c) The Gelfand transformation $\hat{\chi}^{a}$ of a character $\chi^{a}, a \in \Gamma_{+}$on $\bar{\Delta}_{G}$ is the function $\hat{x}^{a}(r g)=\chi^{a}(g) r^{a}$, where $r g \in \bar{\Delta}_{G}$.

(d) The origin $O=(\{0\} \times G) /(\{0\} \times G)$ in $\Delta_{G}$ is a one-point Gleason part for $A_{G}$.

(e) The group $G=b \Delta_{G}$ is the Shilov boundary of $A_{G}$.

(f) Any automorphism $\tau$ of $A_{G}, G \neq \mathbb{T}$ is generated by a pair $(g, \varphi)$ such that $g \in$ $G$ and $\varphi: \Gamma \rightarrow \Gamma$ is an automorphism that preserves $\Gamma_{+}$, that is, $\tau=\tau_{(g, \varphi)}$, where $\tau_{(g, \varphi)}\left(\chi^{a}\right)=\left(\chi^{\varphi(a)}(g)\right) \chi^{\varphi(a)}$. The automorphisms of $A_{G}$ in the case when $G=\mathbb{T}$ are the Möbius transformations of the unit disc.

3. Blaschke inductive limit algebras. Let $\Lambda=\left\{d_{k}\right\}_{k=1}^{\infty}$ be a sequence of natural numbers. Suppose that $m_{k}=\prod_{l=1}^{k} d_{l}, m_{0}=1$, and denote by $\Gamma_{\Lambda}$ the abelian subgroup of $\mathbb{Q}$, that is, generated by the numbers $1 / m_{k}, k \in \mathbb{N}$. The group $\Gamma_{\Lambda}$ can be expressed as the inductive (direct) limit of groups $\mathbb{Z}$, namely

$$
\mathbb{Z}_{1} \stackrel{\zeta_{1}^{2}}{\longrightarrow} \mathbb{Z}_{2} \stackrel{\zeta_{2}^{3}}{\longrightarrow} \mathbb{Z}_{3} \stackrel{\zeta_{3}^{4}}{\longrightarrow} \mathbb{Z}_{4} \stackrel{\zeta_{4}^{5}}{\longrightarrow} \cdots \rightarrow \Gamma_{\Lambda},
$$

where $\zeta_{k}^{k+1}\left(m_{k}\right)=d_{k} \cdot m_{k}, m_{k} \in \mathbb{Z}_{k}=\mathbb{Z}$. The corresponding dual groups form an inverse (projective) sequence of unit circles, whose limit is the compact abelian group $G_{\Lambda}=\widehat{\Gamma}_{\Lambda}$, that is,

$$
\mathbb{T}_{1} \stackrel{\tau_{1}^{2}}{\longleftarrow} \mathbb{T}_{2} \stackrel{\tau_{2}^{3}}{\longleftarrow} \mathbb{T}_{3} \stackrel{\tau_{3}^{4}}{\longleftarrow} \mathbb{T}_{4} \stackrel{\tau_{4}^{5}}{\longleftarrow} \cdots \leftarrow G_{\Lambda} .
$$

Here $\mathbb{T}_{k}=\mathbb{T}$ are unit circles, and $\tau_{k}^{k+1}(z)=\left(\zeta_{k}^{k+1}\right)^{*}(z)=z^{d_{k}}$. Indeed, $\tau_{k}^{k+1}\left(e^{i t m}\right)=$ $e^{i t \zeta_{k}^{k+1}(m)}=e^{i t d_{k} m}=\left(e^{i t m}\right)^{d_{k}}$ for every $e^{i t m} \in \mathbb{T}_{k}=\hat{\mathbb{Z}}_{k}$.

There arises a conjugated inductive system $\left\{A\left(\mathbb{T}_{k}\right), i_{k}^{k+1}\right\}_{k=1}^{\infty}$ of disc algebras $A(\mathbb{T})$ linked by homomorphisms $i_{k}^{k+1}=C_{\tau_{k}^{k+1}}: A\left(\mathbb{T}_{k}\right) \rightarrow A\left(\mathbb{T}_{k+1}\right): i_{k}^{k+1}(f)=f \circ \tau_{k}^{k+1}$, that is, $\left(i_{k}^{k+1}(f)\right)(z)=f\left(z^{d_{k}}\right)$ for $z \in \mathbb{T}_{k+1}$.

Consider the extensions $\tau_{k}^{k+1}(z)=z^{d_{k}}$ on $\overline{\mathbb{D}}_{k}$. The limit of the inverse sequence $\left\{\overline{\mathbb{D}}_{k}, \tau_{k}^{k+1}\right\}, \varliminf_{k \rightarrow \infty}\left\{\overline{\mathbb{D}}_{k}, \tau_{k}^{k+1}\right\}$ is the big $G_{\Lambda}$-disc $\bar{\Delta}_{G_{\Lambda}}=\left([0,1] \times G_{\Lambda}\right)\left(\{0\} \times G_{\Lambda}\right)$ over the 
group $G_{\Lambda}=\hat{\Gamma}_{\Lambda}$. There arises an analogous inductive system $\left\{A\left(\overline{\mathbb{D}}_{k}\right), i_{k}^{k+1}\right\}_{1}^{\infty}$ of algebras $A(\overline{\mathbb{D}}) \cong A(\mathbb{T})$ and connecting homomorphisms $i_{k}^{k+1}: A\left(\overline{\mathbb{D}}_{k}\right) \rightarrow A\left(\overline{\mathbb{D}}_{k+1}\right)$ defined as before by

$$
i_{k}^{k+1}=C_{z^{d_{k}}}, \quad \text { that is, } \quad\left(i_{k}^{k+1}(f)\right)(z)=(f(z))^{d_{k}} .
$$

The elements of the component algebras $A\left(\overline{\mathbb{D}}_{k}\right)$ can be interpreted as continuous functions on $\bar{\Delta}_{G_{\Lambda}}$. The uniform closure

$$
\left[\underset{k \rightarrow \infty}{\lim _{k}}\left\{A\left(\overline{\mathbb{D}}_{k}\right), C_{z} d_{k}\right\}\right]
$$

in $C\left(\bar{\Delta}_{G_{A}}\right)$ of the inductive limit of the system $\left\{A\left(\overline{\mathbb{D}}_{k}\right), C_{z^{d_{k}}}\right\}_{k=1}^{\infty}$, as well as the corresponding restriction algebra $\left[\underline{\lim }_{k \rightarrow \infty}\left\{A\left(\mathbb{T}_{k}\right), C_{z} d_{k}\right\}\right]$ is isometrically isomorphic to the big $G_{\Lambda}$-disc algebra $A_{G_{\Lambda}}$, that is, to the algebra $A\left(\Delta_{G_{\Lambda}}\right)$ of generalized $G_{\Lambda}$-analytic functions on the big $G_{\Lambda}$-disc $\bar{\Delta}_{G_{\Lambda}}$ (see [10]).

In a similar way, if $\left\{K_{l}\right\}_{l=1}^{\infty}$ is a sequence of compact subsets in the complex plane $\mathbb{C}$ with $\tau_{l}^{l+1}\left(K_{l+1}\right)=K_{l}$ for every $l \in \mathbb{Z}$, then the closure of the inductive limit $\underline{\lim }_{l \rightarrow \infty}\left\{A\left(K_{l}\right)\right.$, $\left.C_{z^{d_{k}}}\right\}$ in $C(\mathscr{K})$ is the algebra of generalized $G_{\Lambda}$-analytic functions $A(\mathscr{K})$ on the compact set $\mathscr{K}=\varliminf_{l \rightarrow \infty}\left\{K_{l}, \tau_{l}^{l+1}\right\}$ in the big $G$-plane $\mathbb{C}_{G_{\Lambda}}$ over the group $G_{\Lambda}$ (see [9]).

Consider an inductive sequence of disc algebras

$$
A\left(\mathbb{T}_{1}\right) \stackrel{i_{1}^{2}}{\longrightarrow} A\left(\mathbb{T}_{2}\right) \stackrel{i_{2}^{3}}{\longrightarrow} A\left(\mathbb{T}_{3}\right) \stackrel{i_{3}^{4}}{\longrightarrow} \cdots,
$$

that are linked by the embeddings $i_{k}^{k+1}: A\left(\mathbb{T}_{k}\right) \rightarrow A\left(\mathbb{T}_{k+1}\right)$. We have that $M_{i_{k}^{k+1}\left(A\left(\mathbb{T}_{k}\right)\right)}=$ $\overline{\mathbb{D}}$, and also $\partial\left(i_{k}^{k+1}\left(A\left(\mathbb{T}_{k}\right)\right)\right)=\mathbb{T}$. According to the remarks following Lemma 2.1 there are finite Blaschke products $B_{k}: \mathbb{D} \rightarrow \mathbb{D}$ such that $i_{k}^{k+1}=C_{B_{k}}$ for every $k \in \mathbb{N}$, that is,

$$
i_{k}^{k+1}(f)=C_{B_{k}}(f)=f \circ B_{k},
$$

where $B_{k}(z)$ is a finite Blaschke product.

Let $b=\left\{B_{k}\right\}_{k=1}^{\infty}$ be the sequence of Blaschke products corresponding to $i_{k}^{k+1}$, that is, $C_{B_{k}}(f)=i_{k}^{k+1}(f)$.

Consider the sequence $\Lambda=\left\{d_{k}\right\}_{k=1}^{\infty}$ of orders of Blaschke products $\left\{B_{k}\right\}_{k=1}^{\infty}$ and let $\Gamma_{\Lambda} \subset \mathbb{Q}$ be the group generated by the numbers $1 / m_{k}, m_{k}=\prod_{l=1}^{k} d_{l}, m_{0}=1$, $k=0,1,2, \ldots$ By $\mathscr{T}_{k}$ we denote the standard $d_{k}$-sheeted lifting of the unit circle $\mathbb{T}$ in the Riemann surface $\mathscr{R}_{k}$ of the function $z^{1 / d_{k}}$. Clearly $\mathscr{T}_{k} \cong \mathbb{T}$, and the diagram

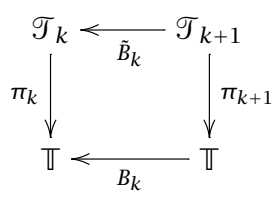

commutes for every $k=0,1,2, \ldots$, where $\pi_{k}$ be the natural covering mapping $\pi_{k}: \mathscr{T}_{k} \rightarrow$ $\mathbb{T}$. The inverse sequence of circles

$$
\mathbb{T}_{1} \stackrel{B_{1}}{\longleftarrow} \mathbb{T}_{2} \stackrel{B_{2}}{\longleftarrow} \mathbb{T}_{3} \stackrel{B_{3}}{\longleftarrow} \mathbb{T}_{4} \stackrel{B_{4}}{\longleftarrow} \cdots \longleftarrow \mathscr{G}_{b}
$$


is isomorphic to the inverse sequence

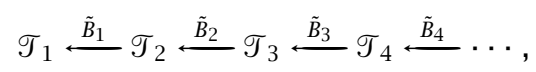

where $\widetilde{B}_{k}$ 's is the natural lifting of $B_{k}$ to $\mathscr{T}_{k}$.

Let again $\tau_{k}^{k+1}(z)=z^{d_{k}}$, and $\tilde{\tau}_{k}^{k+1}(z)$ be the natural lifting of $\tau_{k}^{k+1}$ to $\mathscr{T}_{k}$. Clearly, the diagram

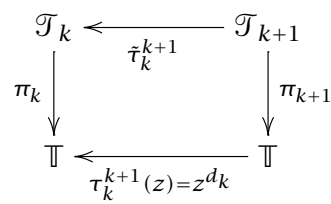

commutes for every $k \in \mathbb{N}$. The inverse sequence (3.9) is (topologically) isomorphic to the sequence

$$
\mathcal{T}_{1} \stackrel{\tilde{\tau}_{1}^{2}}{\longleftarrow} \mathcal{T}_{2} \stackrel{\tilde{\tau}_{2}^{3}}{\longleftarrow} \mathcal{T}_{3} \stackrel{\tilde{\tau}_{3}^{4}}{\longleftarrow} \mathcal{T}_{4} \stackrel{\tilde{\tau}_{4}^{5}}{\longleftarrow} \cdots,
$$

which on its own is isomorphic to the sequence

$$
\mathbb{T}_{1} \stackrel{\tau_{1}^{2}}{\longleftarrow} \mathbb{T}_{2} \stackrel{\tau_{2}^{3}}{\longleftarrow} \mathbb{T}_{3} \stackrel{\tau_{3}^{4}}{\longleftarrow} \mathbb{T}_{4} \stackrel{\tau_{4}^{5}}{\longleftarrow} \cdots \leftarrow G_{\Lambda} .
$$

Consequently, the set $\mathscr{G}_{b}$ from (3.8) is homeomorphic to the group $G_{\Lambda}$. For the dual sequence we get

$$
\mathbb{Z}_{1} \stackrel{\hat{B}_{1}}{\longrightarrow} \mathbb{Z}_{2} \stackrel{\hat{B}_{2}}{\longrightarrow} \mathbb{Z}_{3} \stackrel{\hat{B}_{3}}{\longrightarrow} \cdots \rightarrow \hat{G}_{\Lambda}=\Gamma_{\Lambda} \subset \mathbb{Q} .
$$

We have obtained the following result.

LEMMA 3.1. The inverse limit $\varliminf_{k \rightarrow \infty}\left\{\mathbb{T}_{k},\left.B_{k}\right|_{\mathbb{T}_{k}}\right\}=\mathscr{G}_{b}$ in (3.11) can be equipped with the structure of a compact abelian group isomorphic to $G_{\Lambda}$, where $\Gamma_{\Lambda}=\hat{G}_{\Lambda} \subset \mathbb{Q}$.

Consider an inverse sequence

$$
\overline{\mathbb{D}}_{1} \stackrel{B_{1}}{\longleftarrow} \overline{\mathbb{D}}_{2} \stackrel{B_{2}}{\longleftarrow} \overline{\mathbb{D}}_{3} \stackrel{B_{3}}{\longleftarrow} \overline{\mathbb{D}}_{4} \stackrel{B_{4}}{\longrightarrow} \cdots,
$$

where $b=\left\{B_{k}\right\}_{k=1}^{\infty}$ is a sequence of finite Blaschke products. The inverse limit $\mathscr{D}_{b}=$ $\varliminf_{k \rightarrow \infty}\left\{\overline{\mathbb{D}}_{k}, B_{k}\right\}$ is a Hausdorff compact space. The limit of the adjoint system $\left\{A\left(\overline{\mathbb{D}}_{k}\right)\right.$, $\left.C_{B_{k}}\right\}_{1}^{\infty}$ of disc algebras $A\left(\overline{\mathbb{D}}_{k}\right)$ linked by the homomorphisms

$$
C_{B_{k}}: A\left(\overline{\mathbb{D}}_{k}\right) \longrightarrow A\left(\overline{\mathbb{D}}_{k+1}\right):\left(C_{B_{k}}(f)\right)\left(z_{k+1}\right)=f\left(B_{k}\left(z_{k+1}\right)\right)
$$

is an algebra of functions on $\mathscr{D}_{b}$, and its closure

$$
A(b)=\left[\varliminf_{k \rightarrow \infty}\left\{A\left(\overline{\mathbb{D}}_{k}\right), C_{B_{k}}\right\}\right]
$$

in $C\left(\mathscr{D}_{b}\right)$ is called the Blaschke inductive limit algebra corresponding to the sequence $b=\left\{B_{k}\right\}_{k=1}^{\infty}$ of Blaschke products. Note that $A(b)$ is isometrically isomorphic to the restriction algebra $\left[\underline{\lim }_{k \rightarrow \infty}\left\{A\left(\mathbb{T}_{k}\right), C_{B_{k}}\right\}\right]$. 
Proposition 3.2. Let $b=\left\{B_{k}\right\}_{k=1}^{\infty}$ be a sequence of finite Blaschke products and let $A(b)=\left[\underline{\lim }_{k \rightarrow \infty}\left\{A\left(\mathbb{T}_{k}\right), C_{B_{k}}\right\}\right]$ be the corresponding inductive limit of disc algebras. Then

(i) $A(b)$ is a uniform algebra on the compact set $\mathscr{D}_{b}=\lim _{k \rightarrow \infty}\left\{\overline{\mathbb{D}}_{k}, B_{k}\right\}$.

(ii) The maximal ideal space of $A(b)$ is $\mathscr{D}_{b}$.

(iii) $A(b)$ is a Dirichlet algebra.

(iv) $A(b)$ is a maximal algebra.

(v) The Shilov boundary $\mathscr{G}_{b} \subset \mathscr{D}_{b}$ of $A(b)$ is a group isomorphic to the group $G_{\Lambda}$, whose dual group $\hat{G}_{\Lambda}$ is isomorphic to the group $\Gamma_{\Lambda} \cong \bigcup_{k=0}^{\infty}\left(1 / m_{k}\right) \mathbb{Z} \subset \mathbb{Q}$, where $m_{k}=$ $\prod_{l=1}^{k} d_{l}, m_{0}=1$, and $d_{k}=\operatorname{ord} B_{k}$.

Indeed, under our hypothesis $B_{k}$ maps $\mathbb{T}_{k+1}$ onto $\mathbb{T}_{k}$ and $\mathbb{D}_{k+1}$ onto $\mathbb{D}_{k}$. Since the Shilov boundary of every component algebra $A\left(\mathbb{D}_{k}\right)$ is the unit circle $\mathbb{T}_{k}$, and the maximal ideal space is the disc $\overline{\mathbb{D}}_{k}$, then the properties (i)-(iii) follow from the general results of inductive limits of uniform algebras (e.g., [7]). The maximality of $A(B)$ is a consequence from the following result.

Proposition 3.3. Every inductive limit of maximal algebras is a maximal algebra.

Proof. Let $A=\left[\mathrm{lim}_{\sigma \in \Sigma}\left\{A^{\sigma}, i_{\sigma}^{\tau}\right\}\right]$, where $A^{\sigma}$ are maximal algebras. If $\mathcal{M}_{\sigma}$ is the maximal ideal space of $A^{\sigma}$, then by (i) $\mathcal{M}_{A}=\varliminf_{\sigma}\left\{\mathcal{M}_{\sigma},\left(i_{\sigma}^{\tau}\right)^{*}\right\}$. Fix $h \in C(\mathcal{M}) \backslash A$ and suppose that the algebra $A[h]$ generated by $A$ and $h$ differs from $C\left(\mathcal{M}_{A}\right)$. Clearly, $A[h]=$ $\left[\mathrm{lim}_{\sigma}\left\{A^{\sigma}\left[h_{\sigma}\right],\left(i_{\sigma}^{\tau}\right)^{* *}\right\}\right]$. Let $g \in \varliminf_{\lim _{\sigma}}\left\{A^{\sigma}\left[h_{\sigma}\right],\left(i_{\sigma}^{\tau}\right)^{* *}\right\} \backslash A$, and consider the algebra $\overrightarrow{A[g}] \subset A[h]$. We have that $g=\left\{\left\{g^{\sigma}\right\}_{\sigma \in \Sigma}, g_{\sigma} \in C\left(M_{\sigma}\right)\right\} \in \underline{\lim }_{\sigma \in \Sigma}\left\{C\left(M_{\sigma}\right),\left(i_{\sigma}^{\tau}\right) * *\right\} \backslash A \subset$ $C\left(\mathcal{M}_{A}\right) \backslash A$. Since $i_{\sigma}^{\tau}\left(A^{\sigma}\right) \subset A^{\tau}$ and $g \notin A$, it follows that $\overrightarrow{g^{\sigma} \notin A^{\sigma}}$ for every $\sigma \in \Sigma$. By the maximality we have that $A^{\sigma}\left[g^{\sigma}\right]=C\left(\mu_{\sigma}\right), \sigma \in \Sigma$. Consequently, $A[h] \supset A[g]=$ $\left[\mathrm{lim}_{\sigma}\left\{A^{\sigma}[g],\left(i_{\sigma}^{\tau}\right)^{* *}\right\}\right]=\left[\varliminf_{\sigma}\left\{C\left(\mathcal{M}_{\sigma}\right),\left(i_{\sigma}^{\tau}\right)^{* *}\right\}\right]=C\left(\mathcal{M}_{A}\right)$. This shows that $A$ is a maximal algebra.

We end this section with the following property of big $G$-disc algebras.

THEOREM 3.4. Let $G$ be a compact abelian group whose dual group $\hat{G}$ is isomorphic to a subgroup $\Gamma$ of $\mathbb{R}$. The big $G$-disc algebra $A_{G}$ can be expressed as a Blaschke inductive limit of disc algebras if and only if $\Gamma$ is isomorphic to a subgroup of $\mathbb{Q}$.

Proof. The first part of the theorem follows from Proposition 3.2. Let $\hat{G} \cong \Gamma \subset \mathbb{Q}$ and let $\left\{a_{i}\right\}_{i=1}^{\infty}$ be an enumeration of $\Gamma$. Without loss of generality, we can assume that $a_{1}=1$. Let $\Gamma^{1}=\mathbb{Z}, \Gamma^{2}=\mathbb{Z}+a_{2} \mathbb{Z}, \Gamma^{3}=\mathbb{Z}+a_{2} \mathbb{Z}+a_{3} \mathbb{Z}$, and so forth. Since $\mathbb{Z} \subset \Gamma^{k}$ and $\Gamma^{k}$ is isomorphic to $\mathbb{Z}$, there is a $m_{k} \in \mathbb{N}$, such that $\Gamma^{k}=\left(1 / m_{k}\right) \mathbb{Z}$. By $\Gamma^{k} \subset \Gamma^{k+1}$ we have that $d_{k+1}=\left(m_{k+1}\right) / m_{k} \in \mathbb{Z}$. The inclusion $i_{k}^{k+1}: \Gamma^{k} \hookrightarrow \Gamma^{k+1}$ generates a mapping $\overparen{i_{k}^{k+1}}: \mathbb{Z} \rightarrow \mathbb{Z}$ such that $\widetilde{i_{k}^{k+1}}(1)=d_{k+1}$, thus $\widetilde{i_{k}^{k+1}}(n)=d_{k+1} \cdot n, n \in \mathbb{Z}_{k}$. Clearly, the group

$$
\Gamma \cong \bigcup_{k=1}^{\infty} \frac{1}{m_{k}} \mathbb{Z}=\underset{k \rightarrow \infty}{\lim }\left\{\Gamma^{k}, \widetilde{i_{k}^{k+1}}\right\} \subset \mathbb{Q}
$$

is generated by the numbers $1 / m_{k}, k \in \mathbb{N}$. As we saw at the beginning of this section, the Blaschke inductive limit $\left[\underline{\lim }_{k \rightarrow \infty}\left\{A\left(\mathbb{T}_{k}\right), C_{z^{d_{k}}}\right\}\right]$ corresponding to the sequence $\Lambda=$ $\left\{d_{k}\right\}_{1}^{\infty}$ coincides with the big $\vec{G}_{\Lambda}$-disc algebra $A_{G_{\Lambda}}$. 
THEOREM 3.5. Let $b=\left\{B_{k}\right\}_{k=1}^{\infty}$ be a sequence of finite Blaschke products on $\overline{\mathbb{D}}$ with no more than one critical point $z_{0}^{(k)}$ and such that $B_{k}\left(z_{0}^{(k+1)}\right)=z_{0}^{(k)}$ for $n$ big enough. Then the algebra $A(b)$ is isometrically isomorphic to the big $G_{\Lambda}$-disc algebra $A_{G_{\Lambda}}$, where $\Lambda=\left\{d_{k}\right\}_{k=1}^{\infty}, d_{k}=\operatorname{ord} B_{k}$.

Proof. Without loss of generality, we can suppose that the hypotheses hold for every $n \in \mathbb{N}$. Lemma 2.2 implies that for every Möbius map $\varphi_{k}$ on $\overline{\mathbb{D}}$ with $\varphi_{k}\left(z_{0}^{(k)}\right)=0$ there exist another Möbius map $\varphi_{k+1}$ on $\overline{\mathbb{D}}$ such that the diagram

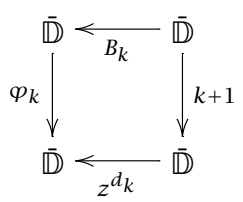

becomes commutative. Hence, $\varphi_{k} \circ B_{k}=\left(\varphi_{k+1}\right)^{d_{k}}$ and $\varphi_{k}\left(z_{0}^{(k)}\right)=0$. Take $\varphi_{0}$ to be the identity on $\overline{\mathbb{D}}$. Lemma 2.2 allows us to define inductively a sequence $\left\{\varphi_{k}\right\}_{k=1}^{\infty}$ of Möbius maps on $\overline{\mathbb{D}}$. Every $\varphi_{k}$ generates an isometric automorphism $C_{\varphi_{k}}$ on $A(\overline{\mathbb{D}})$ such that the conjugate diagram

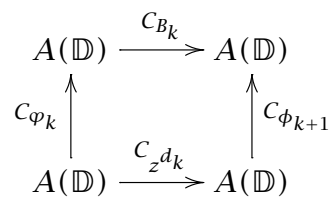

commutes, that is, $C_{B_{k}} \circ C_{\varphi_{k}}=C_{\varphi_{k+1}} \circ C_{z^{d_{k}}}$. Therefore, the inductive sequences

$$
A(\overline{\mathbb{D}}) \stackrel{C_{B_{1}}}{\longrightarrow} A(\overline{\mathbb{D}}) \stackrel{C_{B_{2}}}{\longrightarrow} A(\overline{\mathbb{D}}) \stackrel{C_{B_{3}}}{\longrightarrow} \cdots \rightarrow A(b),
$$

where $C_{B_{k}}(f)=f \circ B_{k}$, and

$$
A(\overline{\mathbb{D}}) \stackrel{C_{z} d_{1}}{\longrightarrow} A(\overline{\mathbb{D}}) \stackrel{C_{z} d_{2}}{\longrightarrow} A(\overline{\mathbb{D}}) \stackrel{C_{z} d_{3}}{\longrightarrow} \cdots \rightarrow A_{G_{\Lambda}},
$$

where $C_{z^{d_{k}}}(f)=f\left(z^{d_{k}}\right)$, are isomorphic. Consequently,

$$
A(b)=\left[\varliminf_{k \rightarrow \infty}\left\{A\left(\overline{\mathbb{D}}_{k}\right), C_{B_{k}}\right\}\right]=\left[\varliminf_{k \rightarrow \infty}\left\{A\left(\overline{\mathbb{D}}_{k}\right), C_{z^{d_{k}}}\right\}\right]=A_{G_{\Lambda}} .
$$

COROLLARY 3.6. If there is a Möbius transformation $\tau$, such that $\left(\tau^{-1} \circ B_{k} \circ \tau\right)(z)=$ $z^{d_{k}} \varphi_{k}(z), k=1,2,3, \ldots$, where $\varphi_{k}$ are Möbius transformations and $d_{k}>1$, then the algebra $A(b)$ is isometrically isomorphic to the big $G$-disc algebra $A_{G}$, where $G$ is the group generated by the numbers $1 / m_{k}, m_{k}=\prod_{l=1}^{k} d_{l}, m_{0}=1, k=0,1,2, \ldots$.

COROLLARY 3.7. If every Blaschke product $B_{k}$ in Theorem 3.5 is a Möbius transformation, then the algebra $A(b)$ is isometrically isomorphic to the disc algebra $A_{\mathbb{Z}}=A(\mathbb{T})$.

Indeed, Theorem 3.5 implies that in this case $A(b)=A_{G_{\Lambda}}$ with $\Lambda=\{1,1, \ldots\}$. Therefore $\Gamma_{\Lambda}=\mathbb{Z}$ and $G_{\Lambda}=\mathbb{T}$. 
As Theorems 3.4 and 3.5 show, certain classes of algebras of $G$-generalized analytic functions can be expressed as inductive limits of disc algebras. Actually, any algebra of generalized $G$-analytic functions can be expressed as inductive limit of an, in general not necessarily countable, inductive spectrum of disc algebras.

4. Annulus type Blaschke algebra $A(b)^{[r, 1]}$. Let $\mathbb{D}^{[r, 1]}=\{z \in \mathbb{C}: r \leq|z| \leq 1\}$, and $b \mathbb{D}^{[r, 1]}=\{z \in \mathbb{C}:|z|=r$ or $|z|=1\}$. Denote by $A\left(\mathbb{D}^{[r, 1]}\right)$ the uniform algebra of continuous functions on $\mathbb{D}^{[r, 1]}$ that are analytic in the interior. Note that $A\left(\mathbb{D}^{[r, 1]}\right)=$ $R\left(\mathbb{D}^{[r, 1]}\right)$, the algebra of continuous rational functions on $\mathbb{D}^{[r, 1]}$. By a well-known result of Bishop, the Shilov boundary of $A\left(\mathbb{D}^{[r, 1]}\right)$ is $b \mathbb{D}^{[r, 1]}$, and the restriction of $A\left(\mathbb{D}^{[r, 1]}\right)$ on $b \mathbb{D}^{[r, 1]}$ is a maximal algebra with $\operatorname{codim} \operatorname{Re}\left(\left.A\left(\mathbb{D}^{[r, 1]}\right)\right|_{b \mathbb{D}^{[r, 1]}}\right)=1$. These results have been extended to the generalized $G$-analytic case in [5]. Namely, let $G$ be a compact abelian group whose dual group is isomorphic to a subgroup $\Gamma$ of $\mathbb{R}$. Let $\Delta_{G}^{[r, 1]}=[r, 1] \times$ $G, 0<r<1$ be the $r$-annulus in the big $G$-disc $\bar{\Delta}_{G}$, and let $R\left(\Delta_{G}^{[r, 1]}\right)$ be the uniform algebra on $\Delta_{G}^{[r, 1]}$, generated by the functions $\hat{x}^{a}, a \in \Gamma$, defined in Section 2 . Then

(a) $\Delta_{G}^{[r, 1]}$ is the maximal ideal space of $R\left(\Delta_{G}^{[r, 1]}\right)$.

(b) $b \Delta_{G}^{[r, 1]}=\{r, 1\} \times G=(\{r\} \times G) \cup(\{1\} \times G)$ is the Shilov boundary of $R\left(\Delta_{G}^{[r, 1]}\right)$.

(c) $R\left(\Delta_{G}^{[r, 1]}\right)$ is a maximal algebra with $\operatorname{codim} \operatorname{Re}\left(\left.R\left(\Delta_{G}^{[r, 1]}\right)\right|_{b \Delta_{G}^{[r, 1]}}\right)=1$.

Consequently, the algebra $R\left(\Delta_{G}^{[r, 1]}\right)$ coincides with the algebra $A\left(\Delta_{G}^{[r, 1]}\right)$ of continuous functions on $\Delta_{G}^{[r, 1]}$ that are locally approximable by generalized $G$-analytic functions in the interior of $\Delta_{G}^{[r, 1]}$.

Let $\Lambda=\left\{d_{k}\right\}_{k=1}^{\infty}$ be a sequence of natural numbers and $\tau_{k}^{k+1}(z)=z^{d_{k}}$. Fix $r \in(0,1]$ and for every $k \in \mathbb{N}$ consider the sets

$$
E_{k}=\mathbb{D}^{\left[r^{\left.1 / m_{k}, 1\right]}\right.}=\left\{z \in \mathbb{C}: r^{1 / m_{k}} \leq|z| \leq 1\right\}=\left(\tau_{1}^{2} \circ \tau_{2}^{3} \circ \cdots \circ \tau_{k}^{k+1}\right)^{-1}\left(\mathbb{D}^{[r, 1]}\right),
$$

where $m_{k}=\prod_{l=1}^{k} d_{l}, m_{0}=1$. Hence, there arises an inverse sequence

$$
\mathbb{D}^{[r, 1]} \stackrel{\tau_{1}^{2}}{\longleftarrow} E_{1} \stackrel{\tau_{2}^{3}}{\longleftarrow} E_{2} \stackrel{\tau_{3}^{4}}{\longleftarrow} E_{3} \stackrel{\tau_{4}^{5}}{\longleftarrow} \cdots
$$

of compact subsets of $\overline{\mathbb{D}}$. Consider the conjugate inductive sequence

$$
A\left(\mathbb{D}^{[r, 1]}\right) \stackrel{C_{z} d_{1}}{\longrightarrow} A\left(E_{1}\right) \stackrel{C_{z} d_{2}}{\longrightarrow} A\left(E_{2}\right) \stackrel{C_{z} d_{3}}{\longrightarrow} \cdots,
$$

where the embeddings $C_{z^{d_{k}}}: A\left(E_{k-1}\right) \rightarrow A\left(E_{k}\right)$ are the composition operators by $z^{d_{k}}$, namely,

$$
\left(C_{z^{d_{k}}} \circ f\right)(z)=f\left(z^{d_{k}}\right) .
$$

Let $G$ denote the compact abelian group whose dual group $\Gamma_{\Lambda}=\hat{G}$ is the subgroup of $\mathbb{Q}$ generated by the numbers $1 / m_{k}, m_{k}=\prod_{l=1}^{k} d_{l}, m_{0}=1, k=0,1,2, \ldots$.

LEMMA 4.1. The uniform algebra $\left[\underline{\lim }_{k \rightarrow \infty}\left\{A\left(E_{k}\right), C_{z^{d_{k}}}\right\}\right]$ is isomorphic to the algebra $A\left(\Delta_{G}^{[r, 1]}\right)$ of $G$-analytic functions on $\overrightarrow{\Delta_{G}^{[r, 1]}}$.

Proof. Let $a_{k}=1 / m_{k}$, where as before $m_{k}=\prod_{l=1}^{k} d_{l}, m_{0}=1$. Consider the algebras $A^{k}\left(\Delta_{G}^{[r, 1]}\right)=\left\{g \circ \hat{x}^{a_{k}}: g \in A\left(E_{k}\right)\right\} \subset A\left(\Delta_{G}^{[r, 1]}\right), k=0,1,2, \ldots$. Clearly, $A^{k}\left(\Delta_{G}^{[r, 1]}\right) \subset A^{k+1}\left(\Delta_{G}^{[r, 1]}\right)$ and $A\left(\Delta_{G}^{[r, 1]}\right)=\left[\bigcup_{k=0}^{\infty} A^{k}\left(\Delta_{G}^{[r, 1]}\right)\right]$. There arises an inductive 
sequence

$$
A^{0}\left(\Delta_{G}^{[r, 1]}\right) \stackrel{j_{0}^{1}}{\longrightarrow} A^{1}\left(\Delta_{G}^{[r, 1]}\right) \stackrel{j_{1}^{2}}{\longrightarrow} A^{2}\left(\Delta_{G}^{[r, 1]}\right) \stackrel{j_{2}^{3}}{\longrightarrow} \cdots \hookrightarrow A\left(\Delta_{G}^{[r, 1]}\right),
$$

where $j_{k}^{k+1}$ is the natural inclusion of $A^{k}\left(\Delta_{G}^{[r, 1]}\right)$ into $A^{k+1}\left(\Delta_{G}^{[r, 1]}\right)$. The inductive sequences (4.3), and (4.5) are isomorphic. Indeed, $\hat{\chi}^{a_{k}}$ maps $\Delta_{G}^{[r, 1]}$ onto $E_{k}$, and the mapping $\varphi_{k}$ defined by $\varphi_{k}\left(g \circ \hat{\chi}^{a_{k}}\right)=g$ maps isometrically and isomorphically $A^{k}\left(\Delta_{G}^{[r, 1]}\right)$ onto $A\left(E_{k}\right)$. In addition, $i_{k+1}^{k+2} \circ \varphi_{k}=\left.\varphi_{k+1}\right|_{A_{k}\left(\Delta_{G}^{[r, 1]}\right)}=\varphi_{k+1} \circ j_{k+1}^{k+2}$, that is, the diagram

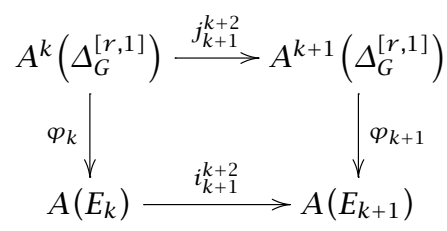

commutes. Therefore (4.3) and (4.5) are two isomorphic sequences, and thus

$$
A\left(\Delta_{G}^{[r, 1]}\right)=\left[\bigcup_{k=0}^{\infty} A_{k}\left(\Delta_{G}^{[r, 1]}\right)\right]=\left[\lim _{k \rightarrow \infty}\left\{A_{k}\left(\Delta_{G}^{[r, 1]}\right), j_{k+1}^{k+2}\right\}\right] \cong\left[\lim _{k \rightarrow \infty}\left\{A\left(E_{k}\right), i_{k+1}^{k+2}\right\}\right] .
$$

Let now $b=\left\{B_{k}\right\}_{k=1}^{\infty}$ be a sequence of finite Blaschke products on $\overline{\mathbb{D}}$ and let $d_{k}=$ $\operatorname{ord} B_{k}$. Define inductively the sets

$$
F_{n}=B_{n}^{-1}\left(F_{n-1}\right)=\left\{z \in \mathbb{C}: B_{n}(z) \in F_{n-1}\right\}=\left(B_{1} \circ B_{2} \circ \cdots \circ B_{n}\right)^{-1}\left(\mathbb{D}^{[r, 1]}\right), \quad F_{0}=\mathbb{D}^{[r, 1]} .
$$

Consider the following conjugate sequences

$$
\begin{aligned}
& \mathbb{D}^{[r, 1]} \stackrel{B_{1}}{\longleftarrow} F_{1} \stackrel{B_{2}}{\longleftarrow} F_{2} \stackrel{B_{3}}{\longleftarrow} F_{3} \stackrel{B_{4}}{\longleftarrow} \cdots \leftarrow \mathscr{D}_{b}^{[r, 1]} \subset \mathscr{D}_{b}, \\
& A\left(\mathbb{D}^{[r, 1]}\right) \stackrel{C_{B_{1}}}{\longrightarrow} A\left(F_{1}\right) \stackrel{C_{B_{2}}}{\longrightarrow} A\left(F_{2}\right) \stackrel{C_{B_{3}}}{\longrightarrow} \cdots,
\end{aligned}
$$

where $\left(C_{B_{k}} \circ f\right)(z)=f\left(B_{k}(z)\right)$.

THEOREM 4.2. If the Blaschke products $B_{n}$ do not have critical points on $F_{n}$ for any $n \in \mathbb{N}$, then $\mathscr{D}_{b}^{[r, 1]} \approx \Delta_{G}^{[r, 1]}$ and the algebra $A(b)^{[r, 1]}=\left[\underline{\lim }_{n \rightarrow \infty}\left\{A\left(F_{n}\right), B_{n}\right\}\right]$ is isometrically isomorphic to the algebra $A\left(\Delta_{G}^{[r, 1]}\right)$.

For the proof we need the following version of a well-known result about Riemann surfaces.

LEMMA 4.3. Suppose that the $d_{k}$-sheeted holomorphic covering $B_{k}: F_{k} \rightarrow F_{k-1}$ does not have critical points, and there exist a biholomorphic mapping $\psi_{k-1}$ from $F_{k-1}$ onto $E_{k-1}$. Then there exist a biholomorphic mapping $\psi_{k}: F_{k} \rightarrow E_{k}$ such that the diagram

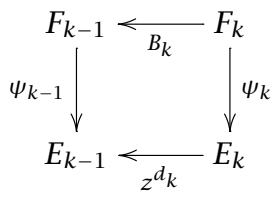

is commutative, that is, $\psi_{k-1} \circ B_{k}=\left(\psi_{k}\right)^{d_{k}}$, where $d_{k}=\operatorname{ord} B_{k}$. 
Proof. The function $z^{d_{k}}$ generates a bijection $\widetilde{z^{d_{k}}}$ from $E_{k}$ onto the $d_{k}$-sheeted covering $\widetilde{E}_{k-1}$ over $E_{k-1}$. Likewise, the map $\psi_{k-1} \circ B_{k}: F_{k} \rightarrow E_{k-1}$ generates a bijection $\left(\psi_{k-1} \circ B_{k}\right)^{\sim}$ from $F_{k}$ to $\widetilde{E}_{k-1}$. Therefore the map $\psi_{k}=\left(\widetilde{z^{d_{k}}}\right)^{-1} \circ\left(\psi_{k-1} \circ B_{k}\right) \sim$ is a bijection from $F_{k}$ onto $E_{k}$. Since all component mappings of $\psi_{k}$ are locally holomorphic, so is $\psi_{k}$.

Proof OF TheOrem 4.2. Let $\psi_{0}$ be the identity map on $\mathbb{D}^{[r, 1]}=E_{0}=F_{0}$. Lemma 4.3 allows us to define inductively biholomorphic mappings $\psi_{k}: F_{k} \rightarrow E_{k}$ for every $k \in \mathbb{N}$ such that $\psi_{k-1} \circ B_{k}=\left(\psi_{k}\right)^{d_{k}}$. Consequently, $\Delta_{G}^{[r, 1]}=\varliminf_{n \rightarrow \infty}\left\{E_{n}, z^{d_{n}}\right\} \approx \varliminf_{n \rightarrow \infty}\left\{F_{n}, B_{n}\right\}$ $=\mathscr{D}_{b}^{[r, 1]} \subset \mathscr{D}_{b}$. The conjugate map $C_{\psi_{k}}$ maps the algebra $A\left(E_{k}\right)$ isometrically and isomorphically onto $A\left(F_{k}\right)$. Hence the inductive sequences (4.3) and (4.10) are isomorphic, and therefore,

$$
A(b)[r, 1]=\left[\varliminf_{k \rightarrow \infty}\left\{A\left(F_{k}\right), C_{B_{k}}\right\}\right]=\left[\varliminf_{k \rightarrow \infty}\left\{A\left(E_{k}\right), z^{d_{k}}\right\}\right] \cong A\left(\Delta_{G}^{[r, 1]}\right) .
$$

In the setting of Theorem 4.2 the listed below properties of the algebra $A(b)^{[r, 1]}$ follow directly from Theorem 4.2, Proposition 3.3, and the results in [6].

(a) The maximal ideal space of the algebra $A(b)^{[r, 1]}$ is homeomorphic to the set $\Delta_{G}^{[r, 1]}$.

(b) The Shilov boundary of $A(b)^{[r, 1]}$ is the set $b \Delta_{G}^{[r, 1]}=\{r, 1\} \times G$.

(c) $A(b)^{[r, 1]}$ is a maximal algebra on its Shilov boundary.

(d) $\operatorname{codim} \operatorname{Re}\left(\left.A(b)^{[r, 1]}\right|_{b \Delta_{G}^{[r, 1]}}\right)=1$.

(e) One-point Gleason parts of $A(b)^{[r, 1]}$ belong to the Shilov boundary $b \Delta_{G}^{[r, 1]}$.

5. Local structure of Blaschke inductive limit algebras. Let $F$ be a closed subset of the unit disc $\mathbb{D}$. Denote by $A(F)$ the algebra of all continuous functions on $F$ that are analytic in the interior of $F$. Recall that $A(F)$ coincides with the uniform closure on $F$ of the restrictions of Gelfand transforms of the elements in $A(\mathbb{T})$ on $F$. That is, $A(F)=\left.\hat{A}(\mathbb{D})\right|_{F}$.

Let $b=\left\{B_{1}, B_{2}, \ldots, B_{n}, \ldots\right\}$ be a sequence of finite Blaschke products on $\mathbb{D}$ and let $0<r<1$. Consider the following compact subsets of $\overline{\mathbb{D}}: D_{n}^{(r)}=B_{n}^{-1}\left(D_{n-1}^{(r)}\right)$, for $n \geq 1$, $D_{0}^{(r)}=\mathbb{D}^{[0, r]}=\{z \in \mathbb{D}:|z| \leq r\}$. There arises an inverse sequence

$$
\mathbb{D}^{[0, r]} \stackrel{B_{1}}{\longleftarrow} D_{1}^{(r)} \stackrel{B_{2}}{\longleftarrow} D_{2}^{(r)} \stackrel{B_{3}}{\longleftarrow} D_{3}^{(r)} \stackrel{B_{4}}{\longleftarrow} \cdots
$$

of subsets of $\mathbb{D}$. The inductive limit

$$
A(b)^{[0, r]}=\left[\underset{n \rightarrow \infty}{\lim _{n \rightarrow \infty}}\left\{A\left(D_{n}^{(r)}\right), C_{B_{n+1}}\right\}\right]
$$

is again a uniform algebra on its maximal ideal space $\varliminf_{k \rightarrow \infty}\left\{D_{n}^{(r)},\left.B_{n+1}\right|_{D_{n}^{(r)}}\right\}=\mathscr{D}_{b}^{[0, r]} \subset$ $\mathscr{D}_{b}$. Every Blaschke product

$$
B(z)=e^{i \theta} \prod_{k=1}^{n}\left(\frac{z-z_{k}}{1-\bar{z}_{k} z}\right), \quad\left|z_{k}\right|<1
$$


of order $n$ generates an $n$-sheeted covering over each simply connected domain $V \subset \mathbb{D}$ that does not contain critical points of $B$. Thus the set $F=B^{-1}(V) \subset \mathbb{D}$ is biholomorphic to the collection of $n$ copies of $V$, that is, $F \cong V \times F_{n}$, where $F_{n}=\{1,2, \ldots, n\}$, and the algebra $A(F)$ is isomorphic to a subalgebra of the algebra

$$
A^{(n)}(V)=A(V) \oplus A(V) \oplus \cdots \oplus A(V) \cong A\left(V \times F_{n}\right),
$$

where $A\left(V \times F_{n}\right)$ is the algebra of all continuous functions $f(z, k)$ on $\bar{V} \times F_{n}$ such that $f(\cdot, k) \in A(V), k=1,2, \ldots, n$. Clearly, $\bar{V} \times F_{n}$ is the set of maximal ideals of the algebra $A(F)$, and $\left.A(F)\right|_{\bar{V} \times\{k\}} \cong A(V)$ for every $k=1,2, \ldots, n$. Hence $A(F) \subset A^{(n)}(V)=$ $A\left(V \times F_{n}\right) \subset C\left(\bar{V} \times F_{n}\right)$.

The space $C\left(F_{n}\right)$ can also be considered as a subalgebra of $A^{(n)}(V)$ consisting of all functions $f \in A^{(n)}(V)$ that are constant on the sets $\bar{V} \times\{k\}, k \in F_{n}$.

Proposition 5.1. Let $b=\left\{B_{1}, B_{2}, \ldots, B_{n}, \ldots\right\}$ be a sequence of finite Blaschke products on $\mathbb{D}$ and let $0<r<1$. Suppose that the set $D_{n}^{(r)}$ does not contain critical points of $B_{n}$ for every $n \in \mathbb{N}$. Then

(i) There is a compact Cantor set $Y$ such that $\mathcal{M}_{A(b)[0, r]}=\mathscr{D}_{b}^{[0, r]}=\varliminf_{k \rightarrow \infty}\left\{D_{n}^{(r)}\right.$, $\left.\left.B_{n}\right|_{D_{n}^{(r)}}\right\}$ is homeomorphic to the Cartesian product $\mathbb{D}^{[0, r]} \times Y$.

(ii) The uniform algebra $A(b)^{[0, r]}$ on $\mathscr{D}_{b}^{[0, r]}$ is isometrically isomorphic to an algebra of functions $f(x, y) \in C\left(\mathbb{D}^{[0, r]} \times Y\right)$, such that $f(\cdot, y) \in A\left(\mathbb{D}^{[0, r]}\right)$ for every $y \in Y$.

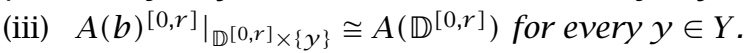

Proof. Consider the inductive sequence

$$
A\left(\mathbb{D}^{[0, r]}\right) \stackrel{C_{B_{1}}}{\longrightarrow} A\left(D_{1}^{(r)}\right) \stackrel{C_{B_{2}}}{\longrightarrow} A\left(D_{2}^{(r)}\right) \stackrel{C_{B_{3}}}{\longrightarrow} \cdots \rightarrow A_{b}^{[0, r]} .
$$

Since the set $D_{m}^{(r)}=B_{m}^{-1}\left(D_{m-1}^{(r)}\right)$ is biholomorphic to $\mathbb{D}^{[0, r]} \times F_{m_{n}}$ for $n \geq 1$, there arises a mapping $j_{k}: \mathbb{D}^{[0, r]} \times F_{m_{k}} \rightarrow \mathbb{D}^{[0, r]} \times F_{m_{k-1}}$ such that the diagram

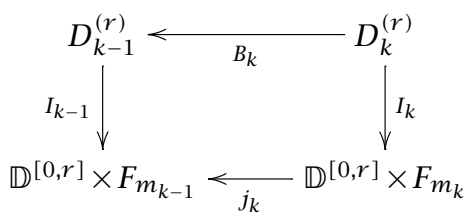

commutes. Note that $j_{k}$ maps $\mathbb{D}^{[0, r]}$ onto $\mathbb{D}^{[0, r]}$, and $F_{m_{k}}$ onto $F_{m_{k-1}}$. Hence, the conjugate diagram

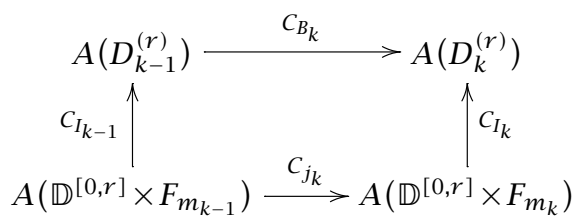

is commutative for every $k \in \mathbb{N}$. Therefore, the inductive sequence (5.5) is isomorphic to the sequence

$$
A\left(\mathbb{D}^{[0, r]}\right) \stackrel{C_{j_{1}}}{\longrightarrow} A\left(\mathbb{D}^{[0, r]} \times F_{m_{1}}\right) \stackrel{C_{j_{2}}}{\longrightarrow} A\left(\mathbb{D}^{[0, r]} \times F_{m_{2}}\right) \stackrel{C_{j_{3}}}{\longrightarrow} \cdots .
$$


Consider the inductive sequence

$$
\mathbb{C} \stackrel{C_{j_{1}}}{\longrightarrow} C\left(F_{m_{1}}\right) \stackrel{C_{j_{2}}}{\longrightarrow} C\left(F_{m_{2}}\right) \stackrel{C_{j_{3}}}{\longrightarrow} \cdots
$$

of restrictions of $A\left(\mathbb{D}^{[0, r]} \times F_{m_{k}}\right)$ on $F_{m_{k}}$. Let $B=\left[\underline{\lim }_{n \rightarrow \infty}\left\{C\left(F_{m_{n}}\right), C_{j_{n+1}}\right\}\right]$. A straightforward check shows that $B$ is a commutative $C^{*}$-algebra. Therefore $B=C(Y)$, where $Y=\varliminf_{n \rightarrow \infty}\left\{F_{m_{n}},\left.j_{n}\right|_{F_{m_{n}}}\right\}$ is a Cantor set. Note that the inductive sequence (5.1) is isomorphic to the sequence

$$
\mathbb{D}^{[0, r]} \stackrel{j_{1}}{\longleftarrow} \mathbb{D}^{[0, r]} \times F_{m_{1}} \stackrel{j_{2}}{\longleftarrow} \mathbb{D}^{[0, r]} \times F_{m_{2}} \stackrel{j_{3}}{\longleftarrow} \mathbb{D}^{[0, r]} \times F_{m_{3}} \stackrel{j_{4}}{\longleftarrow} \cdots \leftarrow \mathbb{D}^{[0, r]} \times Y .
$$

Clearly, the algebra $A(b)^{[0, r]}=\left[\varliminf_{n \rightarrow \infty}\left\{A\left(D_{n}^{(r)}\right), C_{B_{n+1}}\right\}\right] \cong\left[\varliminf_{n \rightarrow \infty}\left\{A\left(\mathbb{D}^{[0, r]} \times F_{m_{n}}\right)\right.\right.$,

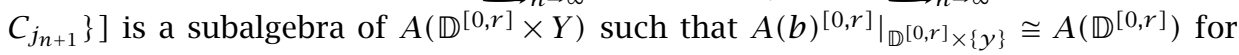
every $y \in Y$.

Note that the set $Y$ here is homeomorphic to $\left\{\left\{y_{n}\right\}_{n=1}^{\infty}, y_{n} \in\left(B_{1} \circ B_{2} \circ \cdots \circ B_{n}\right)^{-1}(0)\right\}$. Since

$$
b \mathscr{D}_{b}^{[0, r]}=\lim _{n \rightarrow \infty}\left\{b D_{n}^{(r)},\left.B_{n}\right|_{b D_{n}^{(r)}}\right\} \approx \mathbb{T}_{r} \times Y,
$$

Proposition 5.1 implies the following corollary.

COROLLARY 5.2. In the setting of Proposition 5.1, the only one-point Gleason parts of the algebra $A(b)^{[0, r]}$ are the points of the Shilov boundary $b \mathscr{D}_{b}^{[0, r]} \approx \mathbb{T}_{r} \times Y$.

Proposition 5.3. Let $b=\left\{B_{1}, B_{2}, \ldots, B_{n}, \ldots\right\}$ be a sequence of finite Blaschke products on $\mathbb{D}$, and let $0<r<1$. Suppose that

(a) For every $n \in \mathbb{N}$ the points of the set $\left(B_{1} \circ B_{2} \circ \cdots \circ B_{n}\right)^{-1}(0)$ are the only singular points for $B_{n}$ in $D_{n}^{(r)}$.

(b) All points in (a) have one and the same order $d_{n}>1$.

Then

(i) There is a compact Cantor set $Y$ such that $\mu_{A(b)}[0, r]=\mathscr{D}_{b}^{[0, r]}=\varliminf_{k \rightarrow \infty}\left\{D_{n}^{(r)}\right.$, $\left.\left.B_{n}\right|_{D_{n}^{(r)}}\right\}$ is homeomorphic to the Cartesian product $\Delta_{G_{\Lambda}}^{[0, r]} \times Y$, where $\Lambda=\left\{d_{k}\right\}_{k=1}^{\infty}$ is the sequence of the orders of $B_{k}$.

(ii) The uniform algebra $A(b)^{[0, r]}$ on $\mathscr{D}_{b}^{[0, r]}$ is isometrically isomorphic to an algebra of functions $f(x, y) \in C\left(\Delta_{G_{\Lambda}}^{[0, r]} \times Y\right)$, such that $f(\cdot, y) \in A\left(\Delta_{G_{\Lambda}}^{[0, r]}\right)$ for every $y \in Y$.

(iii) $\left.A(b)^{[0, r]}\right|_{\Delta^{[0, r]} \times\{y\}} \cong A\left(\Delta_{G_{\Lambda}}^{[0, r]}\right)$ for every $y \in Y$.

Proof. The set $\left(B_{1} \circ B_{2} \circ \cdots \circ B_{n}\right)^{-1}\left(D_{n}^{(r)}\right) \subset \mathbb{D}$ is biholomorphic to the collection of $m_{n}$ copies of $D_{n}^{(r)}$, that is, $F \cong D_{n}^{(r)} \times F_{m_{n}}, F_{m_{n}}=\left\{1,2, \ldots, m_{n}\right\}$. In addition, the algebra $A(F)$ is isomorphic to a subalgebra of the algebra

$$
A^{\left(m_{n}\right)}\left(D_{n}^{(r)}\right)=A\left(D_{n}^{(r)}\right) \oplus A\left(D_{n}^{(r)}\right) \oplus \cdots \oplus A\left(D_{n}^{(r)}\right) \cong A\left(D_{n}^{(r)} \times F_{m_{n}}\right) .
$$

Moreover, $\left.A(F)\right|_{D_{n}^{(r)} \times\{k\}} \cong A\left(D_{n}^{(r)}\right)$ for every $k=1,2, \ldots, m_{n}$. Hence $A(F) \subset A^{\left(m_{n}\right)}\left(D_{n}^{(r)}\right)$ $=A\left(D_{n}^{(r)} \times F_{m_{n}}\right) \subset C\left(D_{n}^{(r)} \times F_{m_{n}}\right)$, while $D_{n}^{(r)} \times F_{m_{n}}$ is the set of maximal ideals of $A(F)$. Consider the space $C\left(F_{m_{n}}\right)$ as a subalgebra of $A^{\left(m_{n}\right)}\left(D_{n}^{(r)}\right)$ consisting all of functions $f \in A^{\left(m_{n}\right)}\left(D_{n}^{(r)}\right)$ that are constant on the sets $D_{n}^{(r)} \times\{k\}, k \in F_{m_{n}}$. As in the proof 
of Proposition 5.1, $B=\left[\lim _{n \rightarrow \infty}\left\{C\left(F_{m_{n}}\right), C_{j_{n+1}}\right\}\right]=C(Y)$, where $Y$ is the Cantor set $\varliminf_{n \rightarrow \infty}\left\{F_{m_{n}},\left.j_{n}\right|_{F_{m n}}\right\}$, and (5.1) is isomorphic to the sequence

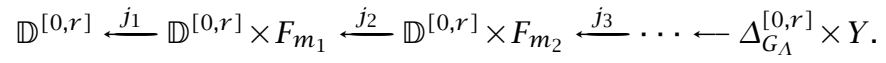

Consequently, the limit $\mathscr{D}_{b}^{[0, r]}$ of the inverse sequence (5.1) is isomorphic to $\Delta_{G_{A}}^{[0, r]} \times$ $Y$. Moreover, the algebra $A(b)^{[0, r]}=\left[\varliminf_{n \rightarrow \infty}\left\{A\left(D_{n}^{(r)}\right), C_{B_{n+1}}\right\}\right]$ is a subalgebra of $C\left(\Delta_{G_{\Lambda}}^{[0, r]} \times Y\right)$ such that $\left.A(b)^{[0, r]}\right|_{\Delta_{G_{\Lambda}}^{[0, r]} \times\{y\}} \cong A\left(\Delta_{G_{\Lambda}}^{[0, r]}\right)$ for every $y \in Y$.

Note that, as before, the set $Y$ is homeomorphic to the set

$$
\left\{\left\{y_{n}\right\}_{n=1}^{\infty}, y_{n} \in\left(B_{1} \circ B_{2} \circ \cdots \circ B_{n}\right)^{-1}(0)\right\} .
$$

Proposition 5.3 and (5.11) imply the following corollary.

COROLLARY 5.4. In the setting of Proposition 5.3, the points of the Shilov boundary $b \mathscr{D}_{b}^{[0, r]} \cong G_{\Lambda} \times Y$ and of the set $\{O\} \times Y$, where $O$ is the origin of the big $G$-disc $\bar{\Delta}_{G_{\Lambda}}$ are the only one-point Gleason parts of the algebra $A(b)^{[0, r]}$.

Corollary 5.4 implies that $A(b)^{[0, r]}$ is isometrically isomorphic to a big $G$-disc algebra if and only if the set $Y$ consists of one point.

COROLLARY 5.5. In the setting of Proposition 5.3 the algebra $A(b)^{[0, r]}$ is isomorphic to a big $G$-disc algebra if and only if every Blaschke product $B_{n}$ has a single critical point $z_{0}^{(n)}$ in $D_{n}^{(r)}$ such that $B_{n}\left(z_{0}^{(n)}\right)=z_{0}^{(n+1)}$ for all $n$ big enough.

6. One-point Gleason parts of Blaschke inductive limit algebras. A celebrated theorem by Wermer states that in every non-one-point Gleason part of the maximal ideal space of a Dirichlet algebra one can embed an analytic disc. Therefore it is of some importance to identify the one-point Gleason parts of an algebra, and especially those of them that do not belong to the Shilov boundary.

Given a sequence of finite Blaschke products $b=\left\{B_{n}\right\}_{n=1}^{\infty}$ on $\overline{\mathbb{D}}$ consider the Blaschke inductive limit algebra $A(b)=\left[\underline{\lim }_{k \rightarrow \infty}\left\{A\left(\overline{\mathbb{D}}_{k}\right), C_{B_{k}}\right\}\right]$ on the compact set $\mathscr{D}_{b}=$ $\varliminf_{k \rightarrow \infty}\left\{\overline{\mathbb{D}}_{k}, B_{k}\right\}$, where $C_{B_{k}}(f)=f \circ B_{k}$. Recall that the Shilov boundary of $A(b)$ is the group $\mathscr{G}_{b}=\varliminf_{k \rightarrow \infty}\left\{\overline{\mathbb{Z}}_{k}, B_{k}\right\}$. Let $\mathscr{B}_{r}$ be the set of all Blaschke products on $\mathbb{D}$ whose zeros are inside the disc $\mathbb{D}_{r}=\{|z|<r\}$, and let $\mathscr{B}_{r}^{0} \subset \mathscr{B}_{r}$ be the set of those products that vanish at 0 . In this section we prove the following theorem.

THEOREM 6.1. Suppose that $B_{n} \in \mathscr{B}_{r}^{0}$ and $\operatorname{ord} B_{n}>1$ for every $n \in \mathbb{N}$. Then $A(b)$ has only one one-point Gleason part in the set $\mathscr{D}_{b} \backslash \mathscr{G}_{b}$.

We proceed with the proof by several lemmas. Given two points $m_{1}$ and $m_{2}$ in $\mathscr{D}_{b}$ consider the Gleason metric

$$
d\left(m_{1}, m_{2}\right)=\sup _{f \in A_{b},\|f\|<1}\left|m_{1}(f)-m_{2}(f)\right| .
$$

LEMMA 6.2. Let $m_{1}=\left(z_{1}, z_{2}, \ldots\right)$, where $z_{k}=B_{k}\left(z_{k+1}\right)$, and $m_{2}=\left(w_{1}, w_{2}, \ldots\right)$, where $w_{k}=B_{k}\left(w_{k+1}\right)$, be the inverse representations of $m_{1}$ and $m_{2} \in \mathscr{D}_{b}$, correspondingly. 
Then

$$
\frac{4 d\left(m_{1}, m_{2}\right)}{4+d^{2}\left(m_{1}, m_{2}\right)}=\lim _{k \rightarrow \infty}\left|\frac{z_{k}-w_{k}}{1-\bar{w}_{k} z_{k}}\right| .
$$

Proof. Let $z_{k}, w_{k} \in \mathbb{D}$ denote the restrictions of $m_{1}$ and $m_{2}$ on $A\left(\overline{\mathbb{D}}_{k}\right)$, respectively. Define

$$
d_{k}\left(m_{1}, m_{2}\right)=\sup _{f \in A\left(\mathbb{\mathbb { D }}_{k}\right),\|f\|<1}\left|m_{1}(f)-m_{2}(f)\right|=d\left(z_{k}, w_{k}\right) .
$$

Since $C_{B_{k}}\left(A\left(\overline{\mathbb{D}}_{k}\right)\right) \subset A\left(\overline{\mathbb{D}}_{k+1}\right)$ and $A_{b}=\bigcup_{1}^{\infty} A\left(\overline{\mathbb{D}}_{k}\right)$ we have

$$
\begin{aligned}
d_{k}\left(m_{1}, m_{2}\right) & \leq d_{k+1}\left(m_{1}, m_{2}\right) \leq d\left(m_{1}, m_{2}\right), \\
d\left(m_{1}, m_{2}\right) & =\lim _{k \rightarrow \infty} d_{k}\left(m_{1}, m_{2}\right) .
\end{aligned}
$$

Note (see [4]) that

$$
\frac{4 d_{k}\left(m_{1}, m_{2}\right)}{4+d_{k}^{2}\left(m_{1}, m_{2}\right)}=\left|\frac{z_{k}-w_{k}}{1-\bar{w}_{k} z_{k}}\right|
$$

Consequently,

$$
\frac{4 d\left(m_{1}, m_{2}\right)}{4+d^{2}\left(m_{1}, m_{2}\right)}=\lim _{k \rightarrow \infty} \frac{4 d_{k}\left(m_{1}, m_{2}\right)}{4+d_{k}^{2}\left(m_{1}, m_{2}\right)}=\lim _{k \rightarrow \infty}\left|\frac{z_{k}-w_{k}}{1-\bar{w}_{k} z_{k}}\right| .
$$

LEMmA 6.3. For every $\varrho \in[0,1]$ let $\alpha(\varrho)=\sup _{\left|z_{0}\right| \leq r,|z| \leq \varrho}\left|\left(z-z_{0}\right) /\left(1-\bar{z}_{0} z\right)\right|$. Then

$$
\max _{|z|<\varrho}|B(z)|<(\alpha(\varrho))^{\operatorname{ord} B}
$$

for every $B \in \mathscr{B}_{r}$.

Proof. By the well-known properties of Möbius transformations, we have that $\alpha(\varrho) \leq 1$ and $\alpha(\varrho)=1$ only if $\varrho=1$. Consequently, if $|z| \leq \varrho$, then for any $B \in \mathscr{B}_{r}$

$$
|B(z)|=\left|\prod_{k=1}^{n}\left(\frac{z-z_{0}}{1-\bar{z}_{0} z}\right)\right| \leq(\alpha(\varrho))^{n} .
$$

Observe that because $B_{n}(0)=0$ for every $n \in \mathbb{N}$, the point $O=(0,0, \ldots)$ belongs to the maximal ideal space $\mathscr{D}_{b}$ of $A(b)$.

Proposition 6.4. Suppose that $B_{n} \in \mathscr{B}_{r}^{0}$ and $\operatorname{ord} B_{n}>1$ for every $n \in \mathbb{N}$. Then $O=(0,0, \ldots)$ is a one-point Gleason part of $A(b)$ in $\mathscr{D}_{b} \backslash \mathscr{G}_{b}$.

Proof. Let $m=\left(z_{1}, z_{2}, \ldots\right)$ be a point in $\mathscr{D}_{b}$ and let $d(O, m)=d$. By (6.2)

$$
\frac{4 d(O, m)}{4+d^{2}(O, m)}=\lim _{n \rightarrow \infty}\left|z_{n}\right|=\frac{4 d}{4+d^{2}}=c \leq 1 .
$$

According to the Schwartz lemma $\left|z_{n}\right|=\left|B\left(z_{n+1}\right)\right|<\left|z_{n+1}\right|$, and hence $\left|z_{n}\right| \leq c$ for every $n \in \mathbb{N}$. Thus,

$$
\left|z_{n}\right|=\left|B_{n}\left(z_{n+1}\right)\right|=\left|z_{n+1}\right|\left|\frac{B_{n}}{z}\left(z_{n+1}\right)\right|<\left|z_{n+1}\right|(\alpha(c))^{\operatorname{ord} B_{n}-1}<c \alpha(c),
$$


and consequently,

$$
c=\lim _{n \rightarrow \infty}\left|z_{n}\right| \leq c \alpha(c) \leq c .
$$

Therefore $\alpha(c)=1$, and thus $1=c=4 d /\left(4+d^{2}\right)$, that is, $d=d(O, m)=2$, that is, $m$ and $O$ belong to different Gleason parts.

It remains to show that $O$ is the only one-point Gleason part of $A(b)$.

LEMMA 6.5. Let $W$ be a simply connected domain such that $\mathbb{D}_{r} \subset W \subset \mathbb{D}$. Let $K=\mathbb{D} \backslash W$ and $K_{B}=\mathbb{D} \backslash B^{-1}(W)$. If the boundary $b W$ of $W$ is a piecewise smooth curve, then the covering map $K_{B} \rightarrow K$ generated by the Blaschke product $B$ does not have singular points.

Proof. Let $z_{0} \in K$. Consider a simply connected domain $\widetilde{W}, W \subset \widetilde{W} \subset \mathbb{D}$ with a piecewise smooth boundary $b \widetilde{W}$ that contains $z_{0}$. As a pre-image of a simply connected domain, $B^{-1}(\widetilde{W})$ also is a simply connected domain with a piecewise smooth boundary $b B^{-1}(\widetilde{W})=B^{-1}(b \widetilde{W})$. Since all zeros of $B$ belong to $\mathbb{D}_{r} \subset W \subset \widetilde{W}$, the Argument Principle for analytic functions implies that every turn along $b B^{-1}(\widetilde{W})$ generates ord $B$ turns along $b \widetilde{W}$. Therefore, $\operatorname{card}\left(B^{-1}\left(z_{0}\right)\right)=\operatorname{ord} B$, that is, $z_{0}$ is not a critical point for $B$.

Proof of Theorem 6.1. Because of Proposition 6.4 it remains to show that the point $O=(0,0, \ldots)$ is the only one-point Gleason part for $A(b)$. Let $m \in \mathscr{D}_{b}, m=$ $\left(z_{1}, z_{2}, \ldots, z_{n}, \ldots\right) \neq O$. As we saw in the proof of Proposition $6.4,\left|z_{n}\right|<\left|z_{k+1}\right|$ for every $n \in \mathbb{N}$, and $\lim _{n \rightarrow \infty}\left|z_{n}\right|=1$. Therefore, without loss of generality we can assume that

$$
\left|z_{1}\right|>r+\varepsilon, \quad \text { where } \varepsilon=\frac{1-r}{2} .
$$

Consider the simply connected domains

$$
\begin{gathered}
W_{n+1}=B_{n}^{-1}\left(W_{n}\right), \quad W_{0}=\mathbb{D}_{r+\varepsilon / 2}, \\
K_{0}=\mathbb{D}^{[0, r+\varepsilon / 2]}, \quad K_{n+1}=B_{n}^{-1}\left(K_{n}\right)=\mathscr{D}_{b} \backslash W_{n+1} .
\end{gathered}
$$

Lemma 6.5 implies that $B_{n}$ has no singularities on $K_{n+1}$. According to Theorem 4.2, $A(b)^{[r, 1]}$ is isomorphic to $A\left(\Delta_{G_{A}}^{[r, 1]}\right)$. Clearly $\mathcal{M}_{A(b)[r, 1]} \subset \mathscr{D}_{b}$, and $\left.A(b)\right|_{\mathcal{M}_{A(b)}[r, 1]}$ is a uniform subalgebra of $A(b)^{[r, 1]}$. The point $m$ belongs to the interior of $\mathcal{M}_{A(b)}^{[r, 1]}$ since $z_{n} \in \operatorname{Int} K_{n}$ for every $n \in \mathbb{N}$.

If we assume that $m$ is the only point in its Gleason part with respect to $A(b)$, then

$$
\sup _{f \in A(b))^{[r, 1],}\|f\|_{\infty}=1}\left|\tilde{f}\left(m_{1}\right)-\tilde{f}(m)\right| \geq \sup _{f \in A(b),\|f\|_{\infty}=1}\left|\tilde{f}\left(m_{1}\right)-\tilde{f}(m)\right|=2
$$

for every $m_{1} \in M_{A(b)[r, 1]}$, that is, $m$ is the only point in its Gleason part for $A(b)^{[r, 1]}$, a contradiction. Hence, $m$ does not belong to any one-point Gleason part of $A(b)^{[r, 1]}$.

COROLlarY 6.6. Let $B \in \mathscr{B}_{r}, B(0) \neq 0$, and $B_{k}(z)=z^{d_{k}} B^{c_{k}}, d_{k}>1$. Then $A(b)$ has only one one-point Gleason part in the set $\mathscr{D}_{b} \backslash \mathscr{G}_{b}$. 
7. Blaschke inductive limit algebras and big $G$-disc algebras. Throughout the previous sections we obtained certain relations between Blaschke inductive limit algebras and big $G$-disc algebras (Theorem 3.4, Corollary 5.5). The main theorem in this section provides necessary and sufficient conditions for a Blaschke inductive limit algebra $A(b)$ to be isometrically isomorphic to a big $G$-disc algebra in the case when all Blaschke products $B_{k}: \mathbb{D} \rightarrow \mathbb{D}$ in the generating $A(b)$ sequence $b=\left\{B_{k}\right\}$ are equal. If this is the case, we will denote the Blaschke inductive limit algebra $\left[\lim _{k \rightarrow \infty}\left\{A(\mathbb{D}), C_{B}\right\}\right]$ by $A(B)$ rather than by $A(b)$.

Proposition 7.1. Let $B$ be a finite Blaschke product with $B(0)=0$. If the Blaschke algebra $A(B)=\left[\varliminf_{B \rightarrow \infty}\left\{A(\mathbb{D}), C_{B}\right\}\right]$, is isometrically isomorphic to a big $G$-disc algebra, then necessarily $\overrightarrow{B(z)}=c z^{n}$, where $c \in \mathbb{C},|c|=1$, and $n \in \mathbb{N}$.

We precede the proof of Proposition 7.1 by several lemmas.

LEMMA 7.2. Consider the defining $A(B)$ inductive sequence

$$
A_{1} \stackrel{C_{B}}{\longrightarrow} A_{2} \stackrel{C_{B}}{\longrightarrow} A_{3} \stackrel{C_{B}}{\longrightarrow} \cdots,
$$

where $B(0)=0$ and $A_{k}=A\left(\mathbb{D}_{k}\right)$. For every $n \in \mathbb{N}$ there exists an automorphism $I_{n}$ : $A(B) \rightarrow A(B)$ such that

$$
I_{n}\left(i_{1}\left(A_{1}\right)\right)=i_{n}\left(A_{n}\right),
$$

where $i_{n}: A_{n} \rightarrow A(B)$ is the natural imbedding.

Proof. We prove the result for $I_{2}$. For $n>2$ the proof follows the same lines. For every $n \in \mathbb{N}$ consider the identity mapping $I_{2}^{n}$ of $A_{n}$ onto $A_{n+1}$. For every $n \in \mathbb{N}$ we have that $I_{2}^{n}\left(\operatorname{id}\left(\mathbb{D}_{n}\right)\right)=\operatorname{id}\left(\mathbb{D}_{n+1}\right),\left\|I_{2}^{n}(f)\right\|=\|f\|$ for every $f \in A_{n}$, and henceforth the diagram

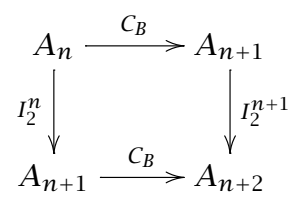

commutes. Consequently, the given inductive sequence is isomorphic to

$$
A_{2} \stackrel{C_{B}}{\longrightarrow} A_{3} \stackrel{C_{B}}{\longrightarrow} A_{4} \stackrel{C_{B}}{\longrightarrow} \cdots .
$$

Clearly, there arises an isometric isomorphism from $\lim _{k \rightarrow \infty}\left\{A(\mathbb{D}), C_{B}\right\}$ onto itself, that can be extended as an automorphism $I_{2}$ of $A(b)=\left[\lim _{k \rightarrow \infty}\left\{A(\mathbb{D}), C_{B}\right\}\right]$ onto itself. It is straightforward to check that $I_{2}$ satisfies (7.2).

COROLLARY 7.3. If $B(0)=0$ then $O=(0,0, \ldots) \in M_{A(B)}$ is a fixed point of the mapping $I_{n}^{*}: M_{A(B)} \rightarrow M_{A(B)}$, that is, conjugate to the automorphism $I_{n}$ from Lemma 7.2.

Proof. Observe that according to Proposition 6.4 and Corollary 6.6, the point $O$ is the only one-point Gleason part of the algebra $A(B)$, that is, outside its Shilov boundary. Since $I_{n}$ is an automorphism, it preserves the structure of the algebra $A(B)$. Therefore the point $I_{n}^{*}(O)$ is also a one-point Gleason part of $A(B)$ out of the Shilov boundary. Hence, $I_{n}^{*}(O)=O$, as claimed. 
The following result is probably well known.

LEMMA 7.4. Let $X$ be a connected compact Hausdorff set and let $\psi_{n} \in C(X)$ be such that $\lim _{n \rightarrow \infty}\left\|\exp \left(\psi_{n}\right)-1\right\|=0$. Then there are $k_{n} \in \mathbb{Z}$ such that the functions $\varphi_{n}=\psi_{n}-2 \pi k_{n} i$ converge uniformly to 0 on $X$.

Proof. If $\psi_{n}=u_{n}+i v_{n}$, then $\exp \left(\psi_{n}\right)=\exp \left(u_{n}\right)\left(\cos v_{n}+i \sin v_{n}\right)$. Вy $\lim _{n \rightarrow \infty}\left\|\exp \left(\psi_{n}\right)-1\right\|=0$ we have that $\exp \left(u_{n}\right) \sin v_{n} \rightarrow 0$ and $\exp \left(u_{n}\right) \cos v_{n} \rightarrow 1$ uniformly on $X$. It follows that $\exp \left(u_{n}\right)$ is a bounded sequence on $X$ and, consequently, $\cos v_{n} \rightarrow 1, \sin v_{n} \rightarrow 0$ uniformly on $X$. The connectedness of $X$ implies that for every $n \in \mathbb{N}$ there is a $k_{n} \in \mathbb{Z}$ such that $\left\|v_{n}-2 \pi k_{n}\right\|<1$. Therefore, $v_{n}-2 \pi k_{n} \rightarrow 0$ because of $\sin v_{n} \rightarrow 0$. Consequently, $\cos \left(v_{n}-2 \pi k_{n}\right) \rightarrow 1$, thus $\exp \left(u_{n}\right) \rightarrow 1$, hence $u_{n} \rightarrow 0$, hence $\varphi_{n}=\psi_{n}-2 \pi k_{n} i \rightarrow 0$ uniformly on $X$, as desired.

Observe that the mapping $i_{1}^{*}: \mu_{A(B)} \rightarrow \overline{\mathbb{D}}$ conjugated to the inclusion $i_{1}: A(\mathbb{D}) \rightarrow$ $A(B)$ maps the Shilov boundary $\partial A(B)=\varphi_{b}$ onto $\mathbb{T}=\partial A(\mathbb{D})$.

LEMMA 7.5. Let $B$ be a finite Blaschke product with $B(0)=0$. If $S$ is an isometric isomorphism from the Blaschke inductive limit algebra $A(B)$ onto a big $G$-disc algebra $A_{G}$, then the set $\left(S \circ i_{1}\right)(A(\mathbb{T}))$ contains necessarily a character $\chi_{1}$ of the group $G=\partial A_{G}$.

Proof. Note that since $\left|\left(S \circ i_{1}\right)(\operatorname{id}(\mathbb{T}))\right|=1$ on $G$, then $\left(S \circ i_{1}\right)(\operatorname{id}(\mathbb{T}))=\chi_{1} \exp (\varphi)$, where $\chi_{1} \in \hat{G}$ and $\varphi \in C(G)$, by the van Kampen theorem [12]. The Arens-Royden theorem (e.g., [3]) assures that $\chi_{1} \in A_{G}$. We show that actually $\chi_{1} \in\left(S \circ i_{1}\right)(A(\mathbb{T}))$.

Let $\chi$ be any fixed element in $\hat{G} \cap A_{G}$. Given an $\varepsilon>0$ one can find an $n \in \mathbb{N}$ so that $d\left(\left(S \circ i_{n}\right)(A(\mathbb{T})), \chi\right)<\varepsilon$, where $d(\cdot, \cdot)$ is the uniform distance in $A_{G} \subset C(G)$. Hence by (7.2) we have

$$
\begin{aligned}
d\left(\left(S \circ i_{1}\right)(A(\mathbb{T})), S I_{n}^{-1} S^{-1} \chi\right) & =d\left(i_{1}(A(\mathbb{T})), I_{n}^{-1} S^{-1} \chi\right) \\
& =d\left(\left(I_{n} \circ i_{1}\right)(A(\mathbb{T})), S^{-1} \chi\right) \\
& =d\left(i_{n}(A(\mathbb{T})), S^{-1} \chi\right) \\
& =d\left(\left(S \circ i_{n}\right)(A(\mathbb{T})), \chi\right)<\varepsilon,
\end{aligned}
$$

where $I_{n}$ is the mapping from Lemma 7.2. As an automorphism of the big $G$-disc algebra $A_{G}$ onto itself, $S I_{n}^{-1} S^{-1}$ maps $\chi$ to a function of type $c \chi_{0}$, where $\chi_{0} \in A_{G}$ is again a character on $G, c \in \mathbb{C},|c|=1$ (see [1]). Therefore, for every $\varepsilon>0$ one can find a character $\left.\chi_{\varepsilon} \in \hat{G} \cap S(A(B))\right|_{G}$ such that

$$
d\left(i_{1}(A(\mathbb{T})), S^{-1} \chi_{\varepsilon}\right)=d\left(\left(S \circ i_{1}\right)(A(\mathbb{T})), \chi_{\varepsilon}\right)<\varepsilon .
$$

By the van Kampen theorem for every $n \in \mathbb{N}$ one can find $m_{n} \in \mathbb{Z}, \psi_{n} \in C(\mathbb{T})$, and $\chi_{1 / n} \in A_{G}$ such that

$$
\left\|\left(\left(S \circ i_{1}\right)\left(\operatorname{id}^{m_{n}}(\mathbb{T})\right) \exp \psi_{n}\right)-\chi_{1 / n}\right\|=\left\|\left(S \circ i_{1}\right)(\operatorname{id}(\mathbb{T}))^{m_{n}} \exp \left(\left(S \circ i_{1}\right) \psi_{n}\right)-\chi_{1 / n}\right\|<\frac{1}{n},
$$

where $i_{1}\left(z^{m_{n}} \exp \left(\psi_{n}\right)\right) \in i_{1}(A(\mathbb{T}))$. Consequently,

$$
\left\|\left(\chi_{1}\right)^{m_{n}} \exp \left(m_{n} \varphi+i_{1}\left(\psi_{n}\right)\right)-\chi_{1 / n}\right\|<\frac{1}{n} .
$$


This can happen only if $\left(\chi_{1}\right)^{m_{n}}=\chi_{1 / n}$. Therefore, we obtain that

$$
\left\|\exp \left(m_{n} \varphi+i_{1}\left(\psi_{n}\right)\right)-1\right\|<\frac{1}{n} .
$$

By Lemma 7.2 we have that the functions $m_{n} \varphi+i_{1}\left(\psi_{n}\right)-2 \pi k_{n} i$ tend uniformly to 0 for some $k_{n} \in \mathbb{Z}$ as $n \rightarrow \infty$. Note that $i_{1}\left(\psi_{n}\right)-2 \pi k_{n} i \in i_{1}(C(\mathbb{T})) \subset C\left(\varphi_{b}\right)=$ $\left[\mathrm{lim}_{k \rightarrow \infty}\left\{C(\mathbb{T}), B^{*}\right\}\right]$. Consequently, $\left\|\varphi+\left(i_{1}\left(\psi_{n}\right)-2 \pi k_{n} i\right) / m_{n}\right\| \rightarrow 0$, and hence $\varphi \in$ $i_{1}(C(\mathbb{T}))$. From $\left(S \circ i_{1}\right)(\operatorname{id}(\mathbb{T}))=\chi_{1} \exp (\varphi)$ we conclude that $\chi_{1} \in\left(S \circ i_{1}\right)(C(\mathbb{T}))$. It remains to show that $\chi_{1} \in\left(S \circ i_{1}\right)(A(\mathbb{T}))$. Suppose that $S^{-1} \chi_{1} \notin i_{1}(A(\mathbb{T})) \subset i_{1}(C(\mathbb{T}))$ and take a $g \in C(\mathbb{T})$ such that $i_{1}(g)=S^{-1} \chi^{a}$. Then $g \notin A(\mathbb{T})$, and the algebra $A_{g}=$ $[A(\mathbb{T}), g]$ on $\mathbb{T}$ generated by $A(\mathbb{T})$ and $g$ equals $C(\mathbb{T})$ by the maximality of the disc algebra $A(\mathbb{T})$. Observe that $i_{1}(C(\mathbb{T}))=i_{1}\left(A_{g}\right)=\left[i_{1}(A(\mathbb{T})),\left(S^{-1} \circ i_{1}\right) g\right]=\left[i_{1}(A(\mathbb{T}))\right.$, $\left.\chi^{a}\right] \subset i_{1}(C(\mathbb{T})) \cap A(B) \mid \varphi_{b}$. However, this contradicts the antisymmetry property of the big $G$-disc algebra $A_{G} \cong A(B)$. We conclude that $S^{-1} \chi_{1} \in i_{1}(A(\mathbb{T}))$, that is, $\chi_{1} \in$ $\left(S \circ i_{1}\right)(A(\mathbb{T}))$.

Proof of Proposition 7.1. Let $i_{1}^{*}: \mathcal{M}_{A(B)} \overline{\mathbb{D}}$ be the conjugate map $i_{1}^{*}\left(z_{1}, z_{2}, \ldots\right)$ $=z_{1}$, where $\left(z_{1}, z_{2}, \ldots\right) \in \varliminf_{k}\{\overline{\mathbb{D}}, B\}$. Note that $i_{1}(O)=0$. By Lemma 7.5 the set $(S \circ$ $\left.i_{1}\right)(A(\mathbb{D})) \cap \hat{G}$ contains a character $\chi_{1} \in \hat{G}$. Let $S^{-1} \chi_{1}=[(h, h \circ B, h \circ B \circ B, \ldots)] \in A(B)$, where $h \in A(\mathbb{T})$. Note that for the Gelfand transform $\widehat{S^{-1} \chi_{1}}$ we have $0=\widehat{S^{-1} \chi_{1}}(O)=$ $\left(i_{1}(h)\right)(O)=h\left(i_{1}^{*}(O)\right)=h(0)$. Suppose that $B\left(z_{0}\right)=0$ for some $z_{0} \in \mathbb{D}$. Then $\widehat{S^{-1} \chi_{1}}\left(0, z_{0}, \ldots\right)=h(0)=0$, and therefore $\left(0, z_{0}, \ldots\right)=O$ since $O$ is the only zero of $\widehat{S^{-1} \chi_{1}}$ in $\mu_{A(B)}$. Hence, $z_{0}=0$, that is, 0 is the only zero of the Blaschke product $B$. Consequently, $B(z)=c z^{m}$ for some $m \in \mathbb{N}, c \in \mathbb{C},|c|=1$.

Theorem 3.5 and Proposition 7.1 imply the following result.

THEOREM 7.6. Let $B$ be a finite Blaschke product on $\mathbb{D}$. The Blaschke inductive limit algebra $A(B)$ is isometrically isomorphic to a big $G$-disc algebra if and only if $B(z)$ is conjugate to some power $z^{m}$ of $z$, that is, if and only if there is an $m \in \mathbb{N}$ and a Möbius transformation $\tau: \mathbb{D} \rightarrow \mathbb{D}$ such that $\left(\tau^{-1} \circ B \circ \tau\right)(z)=z^{m}$.

8. Inductive limits of algebras $H^{\infty}$. Consider the inverse sequence

$$
\mathbb{D}_{1} \stackrel{I_{1}}{\longleftarrow} \mathbb{D}_{2} \stackrel{I_{2}}{\longleftarrow} \mathbb{D}_{3} \stackrel{I_{3}}{\longleftarrow} \mathbb{D}_{4} \stackrel{I_{4}}{\longleftarrow} \cdots,
$$

where $\mathbb{D}_{k}=\mathbb{D}$ and $I=\left\{I_{1}, I_{2}, \ldots, I_{k}, \ldots\right\}$ is a sequence of non-constant inner functions on $\mathbb{D}$. The limit of the inverse sequence $(8.1)$ we denote by $\mathscr{D}_{I}$. The inductive limit $\lim _{k \rightarrow \infty}\left\{H_{k}^{\infty}, I_{k}^{*}\right\}_{1}^{\infty}$ of the adjoint inductive sequence

$$
H_{1}^{\infty} \stackrel{I_{1}^{*}}{\longrightarrow} H_{2}^{\infty} \stackrel{I_{2}^{*}}{\longrightarrow} H_{3}^{\infty} \stackrel{I_{3}^{*}}{\longrightarrow} \cdots
$$

of algebras $H_{k}^{\infty}=H^{\infty}(\mathbb{D})$, where $I_{k}^{*}(f)=f \circ I_{k}$, is a subalgebra of $B C\left(\mathscr{D}_{I}\right)$, the algebra of bounded continuous functions on the set $\mathscr{D}_{I}$. The closure $H_{(I)}^{\infty}$ of $\underline{\lim }_{\mathrm{k} \rightarrow \infty}\left\{H^{\infty}, I_{k}^{*}\right\}$ in $B C\left(\mathscr{D}_{I}\right)$ is a uniform algebra. We call its elements I-hyper-analytic functions on $\mathscr{D}_{I}$.

Recall that according to the classical corona theorem for the space $H^{\infty}$ (Carleson, [2]), given $f_{1}, \ldots, f_{k}$, functions in $H^{\infty}$ with $\sum_{j=1}^{k}\left|f_{j}\right| \geq \sigma>0$ on $\mathbb{D}$, there exist functions 
$g_{1}, \ldots, g_{k}$ in $H^{\infty}$ such that $\sum_{j=1}^{k} f_{j} g_{j}=1$ on $\mathbb{D}$. If $\left\|f_{j}\right\|_{\infty} \leq 1$, then $g_{j}$ can be chosen to satisfy the estimates $\left\|g_{j}\right\| \leq C(k, \sigma)$ for some constant $C(k, \sigma)>0$.

Here we consider and solve the corona problem for the algebra $H_{(I)}^{\infty}$.

THEOREM 8.1. If $f_{1}, f_{2}, \ldots, f_{n},\left\|f_{j}\right\| \leq 1$, are I-hyper-analytic functions on $\mathscr{D}_{I}$ for which

$$
\left|f_{1}(x)\right|+\cdots+\left|f_{n}(x)\right| \geq \delta>0 \quad \text { for each } x \in \mathscr{D}_{I},
$$

then there is a constant $K(n, \delta)$ and I-hyper-analytic functions $g_{1}, \ldots, g_{n}$ on $\mathscr{D}_{I}$ with $\left\|g_{j}\right\| \leq K(n, \delta)$, such that the equality

$$
f_{1}(x) g_{1}(x)+\cdots+f_{n}(x) g_{n}(x)=1
$$

holds for every point $x$ in the set $\mathscr{D}_{I}$.

Observe that the adjoint mappings $I_{j}^{*}: H_{j}^{\infty} \rightarrow H_{j+1}^{\infty}$ are isometric isomorphisms; and so are the mappings $\iota_{j}^{k}: H_{j}^{\infty} \rightarrow H_{k}^{\infty}$ defined by $\iota_{j}^{k}=I_{j}^{*} \circ I_{j+1}^{*} \circ \cdots \circ I_{k}^{*}$. Because of $\left(I_{j}^{*}(f)\right)(z)=f\left(I_{j}(z)\right), z \in \mathbb{D}_{j+1}$ for every $j \in \mathbb{N}$ and $f \in H_{j}^{\infty}$, we have that $\left(\iota_{j}^{k}(f)\right)(z)=$ $f\left(I_{j} \circ I_{j+1} \circ \cdots \circ I_{k}\right)(z)$, where $z \in \mathbb{D}_{k+1}$. Consequently, every component space $H_{j}^{\infty}$ can be embedded isometrically and isomorphically into $\lim _{k \rightarrow \infty}\left\{H^{\infty}, I_{k}^{*}\right\} \subset H_{(I)}^{\infty}$ via a natural mapping $\iota_{j}: H_{j}^{\infty} \rightarrow H_{(I)}^{\infty}$ (see [7]). Moreover, if $z^{*} \in \mathbb{D}_{j}$, then $f\left(z^{*}\right)=\left(\iota_{j}(f)\right)\left(x^{*}\right)$, where $x^{*} \in \mathscr{D}_{I}$ is defined as $x^{*}=\left(z_{1}, z_{2}, \ldots, z_{j}, \ldots\right)$ with $z_{j}=z^{*}$ and $I_{n}\left(z_{n+1}\right)=z_{n}$ for $n \geq j$.

Proof. Without loss of generality we can assume that $\left\|f_{j}\right\| \leq 1 / 2$ for all $f_{j} \in H_{(I)}^{\infty}$ in (8.3) and that $\delta \leq 1 / 2$. Let $C(n, \delta / 2)$ be the corresponding Carleson's constant and let $c=\max \{1, C(n, \delta / 2)\}$. By the definition of the space $H_{(I)}^{\infty}$ there are integers $n_{j} \in \mathbb{N}$ and functions $\tilde{f}_{j} \in H_{n_{j}}^{\infty}$, such that

$$
\left\|f_{j}-\iota_{n_{j}}\left(\tilde{f}_{j}\right)\right\|_{\infty}=\sup _{x \in \mathscr{I}_{I}}\left|f_{j}(x)-\left(\iota_{n_{j}}\left(\tilde{f}_{j}\right)\right)(x)\right|<\frac{\delta}{2 c n}, \quad j=1, \ldots, n .
$$

We may assume (by considering $\iota_{n_{j}}^{m}\left(\tilde{f}_{j}\right)$ instead of $\left.\tilde{f}_{j}\right)$ that all $\tilde{f}_{j} \in H_{m}^{\infty}$ for some $m \geq$ $n_{j}, j=1,2, \ldots, n$. By (8.3) for every $z^{*} \in \mathbb{D}$ we have

$$
\begin{aligned}
\left|\tilde{f}_{1}\left(z^{*}\right)\right|+\cdots+\left|\tilde{f}_{n}\left(z^{*}\right)\right| & =\left|\left(\iota_{m}\left(\tilde{f}_{1}\right)\right)\left(x^{*}\right)\right|+\cdots+\left|\left(\iota_{m}\left(\tilde{f}_{n}\right)\right)\left(x^{*}\right)\right| \\
& \geq \sum_{j=1}^{n}\left|f_{j}\left(x^{*}\right)\right|-\sum_{j=1}^{n}\left|f_{j}\left(x^{*}\right)-\left(\iota_{m}\left(\tilde{f}_{j}\right)\right)\left(x^{*}\right)\right| \\
& \geq \delta-\frac{\delta}{2 c} \geq \frac{\delta}{2}>0
\end{aligned}
$$

where as before $x^{*}=\left(z_{1}, z_{2}, \ldots, z_{m}, \ldots\right)$ with $z_{m}=z^{*}$ and $I_{\tilde{n}}\left(z_{n+1}\right)=z_{n}$ for $n \geq$ $m$. Consequently, for the bounded analytic functions $\tilde{f}_{1}, \ldots, \widetilde{f}_{n}$ on $\mathbb{D}$ we have that $\left|\tilde{f}_{1}\right|+\cdots+\left|\tilde{f}_{n}\right| \geq \delta / 2>0$ on $\mathbb{D}$. In addition,

$$
\left\|\tilde{f}_{j}\right\|_{\infty}=\left\|\iota_{m}\left(\tilde{f}_{j}\right)\right\|_{\infty} \leq\left\|f_{j}\right\|_{\infty}+\left\|f_{j}-\iota_{m}\left(\tilde{f}_{j}\right)\right\|_{\infty} \leq\left\|f_{j}\right\|_{\infty}+\frac{\delta}{2 c n} \leq 1
$$


According to the corona theorem for $H^{\infty}$ there exist functions $h_{1}, \ldots, h_{n} \in H^{\infty}$ with $\left\|h_{j}\right\|_{\infty} \leq C(n, \delta / 2) \leq c$ such that $\tilde{f}_{1} h_{1}+\cdots+\tilde{f}_{n} h_{n}=1$ on $\mathbb{D}$. Hence,

$$
\begin{aligned}
1 & =\left(\tilde{f}_{1} h_{1}+\cdots+\tilde{f}_{n} h_{n}\right)\left(z^{*}\right)=\iota_{m}\left(\tilde{f}_{1} h_{1}+\cdots+\tilde{f}_{n} h_{n}\right)\left(x^{*}\right) \\
& =\left(\iota_{m}\left(\tilde{f}_{1}\right) \iota_{m}\left(h_{1}\right)+\cdots+\iota_{m}\left(\tilde{f}_{n}\right) \iota_{m}\left(h_{n}\right)\right)\left(x^{*}\right)
\end{aligned}
$$

on $\mathscr{D}_{I}$, and $\left\|\iota_{m}\left(h_{j}\right)\right\|_{\infty}=\left\|h_{j}\right\|_{\infty} \leq c$. Note that while the function

$$
F=f_{1} \iota_{m}\left(h_{1}\right)+\cdots+f_{n} \iota_{m}\left(h_{n}\right) \in H_{(I)}^{\infty}
$$

may not be identically equal to 1 on $\mathscr{D}_{I}$, it is invertible in $H_{I}^{\infty}$. Indeed,

$$
\begin{aligned}
\|1-F\|_{\infty} & =\left\|\sum_{j} \iota_{m}\left(\tilde{f}_{j}\right) \iota_{m}\left(h_{j}\right)-\sum_{j} f_{j} \iota_{m}\left(h_{j}\right)\right\|_{\infty} \\
& \leq \sum_{j}\left\|\iota_{m}\left(\tilde{f}_{j}\right)-f_{j}\right\|_{\infty}\left\|\iota_{m}\left(h_{j}\right)\right\|_{\infty} \leq \frac{\delta}{2 c n} c n=\frac{\delta}{2}<1 .
\end{aligned}
$$

Now the identity $f_{1} g_{1}+\cdots+f_{n} g_{n}=1$ holds on $\mathscr{D}_{I}$ with $g_{j}=\iota_{m}\left(h_{j}\right) / F \in H_{(I)}^{\infty}, j=$ $1, \ldots, n$. Note that $\left\|F^{-1}\right\|_{\infty} \leq 1 /(1-\delta / 2)=2 /(2-\delta)$, since $|F(x)| \geq 1-\delta / 2$ on $\mathscr{D}_{I}$ according to (8.10). Hence,

$$
\left\|g_{j}\right\|_{\infty} \leq\left\|\iota_{m}\left(h_{j}\right)\right\|_{\infty}\left\|F^{-1}\right\|_{\infty} \leq \frac{2 c}{2-\delta}=\frac{2 \max \{1, C(n, \delta / 2)\}}{2-\delta} .
$$

The proof is completed by choosing $K(n, \delta)=2 \max \{1, C(n, \delta / 2)\} /(2-\delta)$.

Consider the particular case when $I=\left\{z^{2}, z^{3}, \ldots, z^{n+1}, \ldots\right\}$. The corresponding set $\mathscr{D}_{I}$ then coincides with the open big disc $\Delta_{G}$ over the compact abelian group $G=\widehat{\mathbb{Q}}$ (e.g., [11]), and the algebra $H_{(I)}^{\infty}$ coincides with the set $H_{G}^{\infty}$ of hyper-analytic functions, introduced in [8]. In this case the result in Theorem 8.1 reduces to the corona theorem for $H_{G}^{\infty}$ with estimates, which straightens the result in [8].

ACKNOWLEDGEMENTS. The authors acknowledge with gratitude the support for this paper by an NSF Cooperative International Research Grant with Russia. Thanks are due also to the Mathematisches Forschungsinstitut Oberwolfach, Germany, and the International Banach Center in Warsaw, Poland, where part of the work on this paper was performed, for their hospitality.

\section{REFERENCES}

[1] R. Arens and I. M. Singer, Generalized analytic functions, Trans. Amer. Math. Soc. 81 (1956), 379-393. MR 17,1226e. Zbl 078.10902.

[2] L. Carleson, Interpolations by bounded analytic functions and the corona problem, Ann. of Math. (2) 76 (1962), 547-559. MR 25\#5186. Zbl 112.29702.

[3] T. W. Gamelin, Uniform Algebras, 2nd ed., Chelsea, New York, 1984.

[4] J. B. Garnett, Bounded Analytic Functions, Pure and Applied Mathematics, vol. 96, Academic Press, New York, 1981. MR 83g:30037. Zbl 469.30024.

[5] S. A. Grigoryan, Maximal algebras of generalized analytic functions, Izv. Akad. Nauk Armyan. SSR Ser. Mat. 16 (1981), no. 5, 358-365 (Russian). MR 84d:46081. 
[6] _ Generalized analytic functions, Uspekhi Mat. Nauk 49 (1994), no. 2(296), 342, [translated in Russian Math. Surveys 49 (1994), no. 2, 1-40. Zbl 00764123]. MR 95h:43006.

[7] G. M. Leibowitz, Lectures on Complex Function Algebras, Scott, Foresman and Co., Illinois, 1970. MR 55\#1072. Zbl 219.46037.

[8] T. V. Tonev, The algebra of bounded hyperanalytic functions on the big disc has no corona, Analytic Functions (Proc. Seventh Conf., Kozubnik, 1979), Lecture notes in Math., vol. 798, Springer, Berlin, 1980, pp. 435-438. MR 81h:46058. Zbl 451.30040.

[9] _ Generalized-analytic functions - recent results, C. R. Acad. Bulgare Sci. 34 (1981), no. 8, 1061-1064. MR 83d:30057. Zbl 482.30036.

[10] - Some results of classical type about generalized analytic functions, PLISKA Stud. Math. Bulgar. 4 (1981), 3-9. MR 83b:30050. Zbl 488.30038.

[11] _ Big-Planes, Boundaries and Function Algebras, North-Holland Mathematics Studies, vol. 172, North-Holland Publishing, Amsterdam, 1992. MR 93h:46065. Zbl 755.46020.

[12] E. R. van Kampen, On almost periodic functions of constant absolute values, J. London Math. Soc. 12 (1937), 3-6. Zbl 016.30402.

S. A. Grigoryan: Chebotarev Institute of Mathematics, Kazan State University, KAZAN, RUSSIA

T. V. TONEV: DePARTMENT OF MATHEMATICAL SCIENCES, UNIVERSITY OF MONTANA-MisSOULA, MISSOULA, MT 59812-0864, USA

E-mail address: ma_tt@se7way.umt.edu 


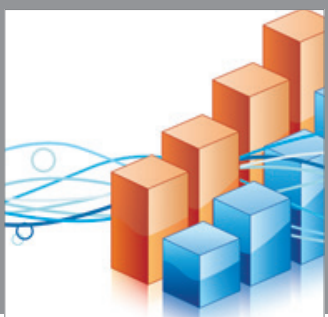

Advances in

Operations Research

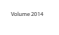

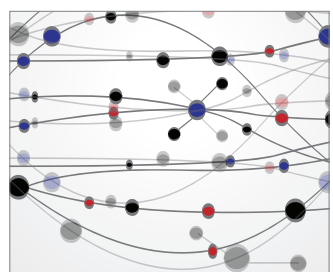

\section{The Scientific} World Journal
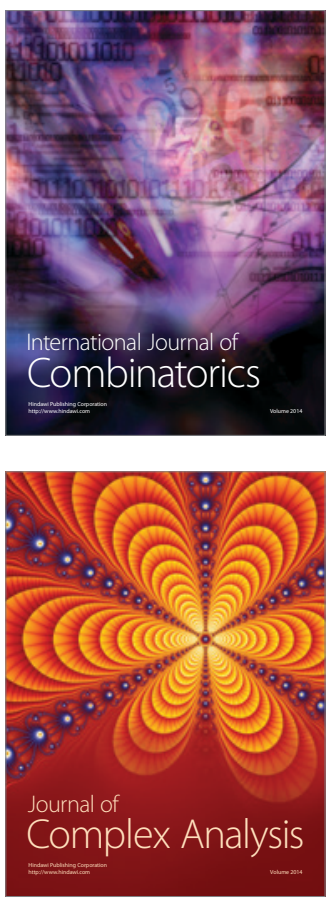

International Journal of

Mathematics and

Mathematical

Sciences
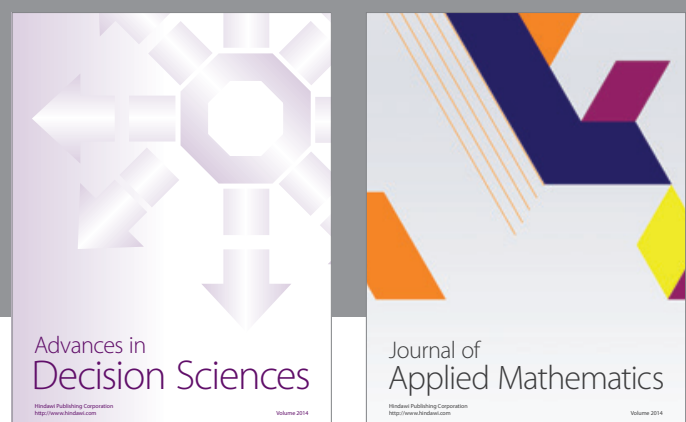

Journal of

Applied Mathematics
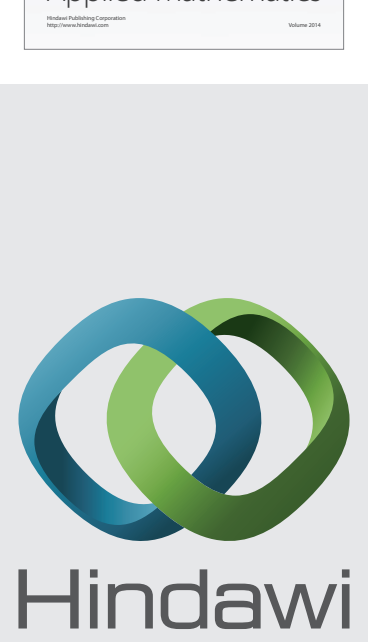

Submit your manuscripts at http://www.hindawi.com
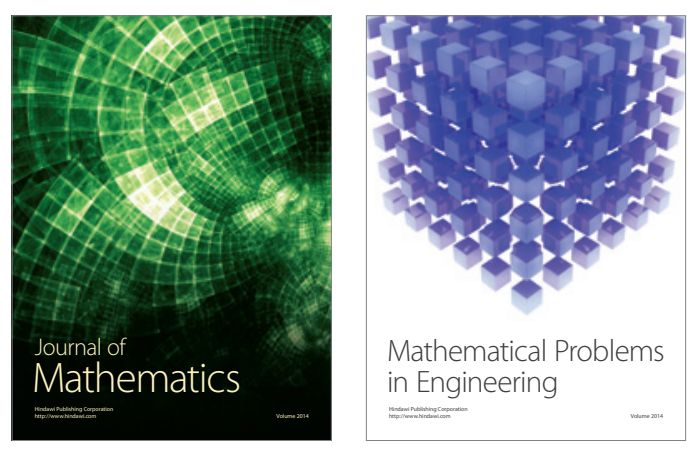

Mathematical Problems in Engineering
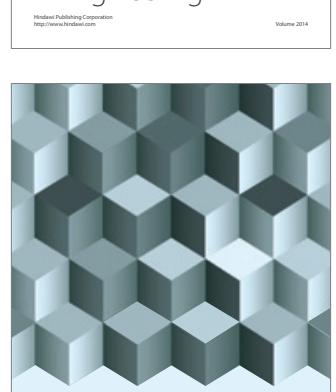

Journal of

Function Spaces
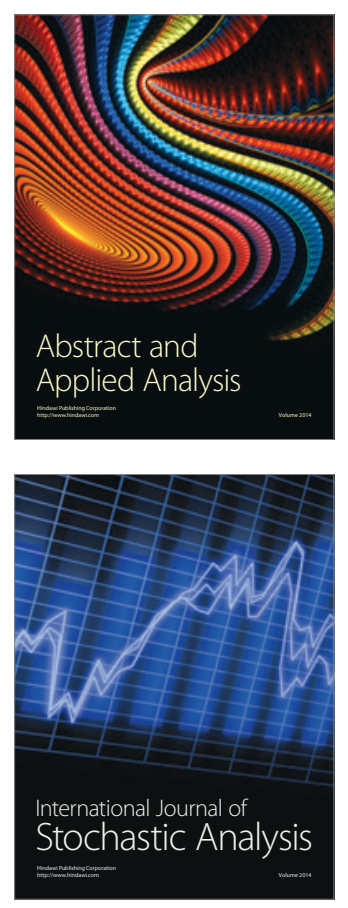

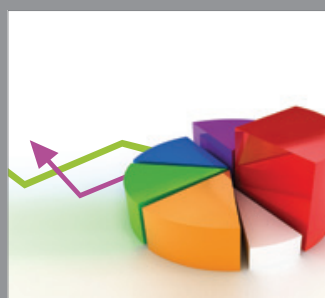

ournal of

Probability and Statistics

Promensencen
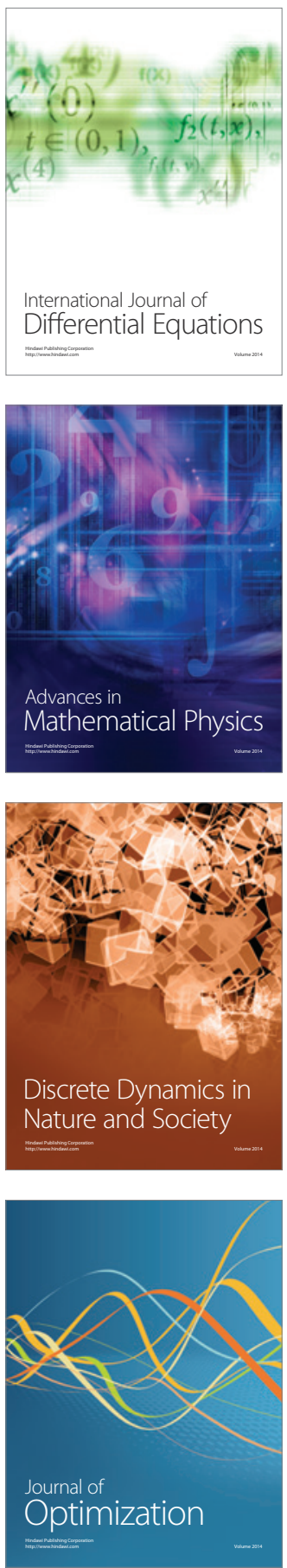